\title{
FOURIER ANALYSIS FOR DENJOY-CARLEMAN CLASSES ON THE TORUS
}

\author{
Bruno de Lessa Victor \\ Universidade Federal do Paraná, Curitiba, Departamento de Matemática \\ PR 82590-300, Brazil; brunodelessa@gmail.com
}

\begin{abstract}
In the present article, we develop Fourier series for a family of classes of Romieu type of ultradifferentiable functions and ultradistributions on the torus, usually known as DenjoyCarleman classes. In this setting, as applications, we extend the Greenfield-Wallach Theorem and, through a conjugation, we characterize global hypoellipticity for a class of systems of real vector fields of tube type.
\end{abstract}

\section{Introduction}

There has been a great development in the field of global analysis in the last decades, especially for problems on the torus $\mathbf{T}^{N}=\mathbf{R}^{N} / 2 \pi \mathbf{Z}^{N}$. An immense amount of results concerning solvability and hypoellipticity for operators and systems (see for instance [AJ, BCP, DGY, WC] and references therein) has been obtained in a variety of frameworks.

Two of those frameworks that stand out are the spaces of real analytic and Gevrey functions (see for example [AZ, Ber, Him, Tar] and references therein). In this context, one of the most important tools is the characterization of ultradistributions and functions via their Fourier series (see for instance [AP, BCCJ, BGS, CC, HP, JKM, PZ]).

An important fact regarding Gevrey spaces is that they generalize the concept of real analytic functions (recall that $\mathcal{G}^{1}\left(\mathbf{T}^{N}\right)=C^{\omega}\left(\mathbf{T}^{N}\right)$ ) and allow us to better understand the gap between smooth and real analytic functions. Nevertheless, they are not sufficient in that sense; In fact, it is possible to show that

$$
C^{\omega}\left(\mathbf{T}^{N}\right) \subsetneq \bigcap_{s>1} \mathcal{G}^{s}\left(\mathbf{T}^{N}\right) .
$$

Furthermore there exist subspaces of $C^{\infty}\left(\mathbf{T}^{N}\right)$ whose elements are nowhere analytic and satisfy the following condition:

$$
x_{0} \in \mathbf{T}^{N} \text { and } D^{\alpha} f\left(x_{0}\right)=0, \forall \alpha \in \mathbf{N}_{0}^{N}, \Rightarrow f \equiv 0,
$$

a property that does not hold for any $\mathcal{G}^{s}\left(\mathbf{T}^{N}\right)$ with $s>1$.

Those facts lead us to the study of Denjoy-Carleman classes; in few words, given a sequence of positive numbers $\left\{m_{n}\right\}_{n \in \mathbf{N}_{0}}$, we work with the space of the functions $f \in C^{\infty}\left(\mathbf{T}^{N}\right)$ such that

$$
\left|D^{\alpha} f(x)\right| \leq C \cdot h^{|\alpha|} \cdot m_{|\alpha|} \cdot|\alpha| !, \quad \forall x \in \mathbf{T}^{N}, \forall \alpha \in \mathbf{N}_{0}^{N},
$$

which provides a much wider range of classes of ultradifferentiable functions.

https://doi.org/10.5186/aasfm.2021.4655

2020 Mathematics Subject Classification: Primary 35-02, 42A16, 35H10.

Key words: Ultradifferentiable classes, Fourier series, global hypoellipticity.

This study was financed in part by the Coordenação de Aperfeiçoamento de Pessoal de Nível Superior - Brasil (CAPES) - Finance Code 001. 
Once we have highlighted the importance of Denjoy-Carleman classes and Fourier analysis, the interest in the development of the latter's techniques in the former's context naturally shows up. That is the principal goal of the present work.

In Section 2, we describe our classes of ultradiferentiable functions, starting with the definition of weight sequences, which characterize the growth of functions and its derivatives. Important examples are presented and we make a brief comparison with the sequences defined by Komatsu in [K1].

Next we endow those classes with a inductive limit topology, which make them DFS spaces and allows us to define the spaces of ultradistributions. In Section 4 we develop Fourier Series for functions and ultradistributions, characterizing both in terms of the decay of their Fourier coefficients.

Finally, Section 5 is destined to the study of global hypoellipticity in the DenjoyCarleman setting. It is divided in three subsections: in the first one it is proved for any class an extension of the Greenfield Theorem [Gr] for systems with constant coefficients. Next we prove a version of the Greenfield-Wallach Theorem [GW] and generalize a construction done in [GPY] which exhibits vector fields that "separate classes" in terms of global hypoellipticity. In the last subsection, inspired in works like $[\mathrm{BCM}],[\mathrm{BP}]$ and $[\mathrm{Hou}]$, we characterize global hypoellipticity for a class of systems of real vector fields of tube type through a conjugation to a system of constant coefficients.

\section{Denjoy-Carleman classes on torus}

In this section we consider a class of weight sequences and give some examples. We also prove conditions which they satisfy and how those properties have impact on the related classes of functions.

Definition 2.1. A weight sequence is a sequence of positive real numbers $\mathscr{M}=$ $\left\{m_{n}\right\}_{n \in \mathbf{N}_{0}}$ satisfying the following conditions:

1. $m_{0}=m_{1}=1 ; \quad$ (initial conditions)

2. $m_{n}^{2} \leq m_{n-1} \cdot m_{n+1}, \quad \forall n \in \mathbf{N} ; \quad$ (logarithmic convexity)

3. $\sup _{j, k}\left(\frac{m_{j+k}}{m_{j} \cdot m_{k}}\right)^{1 /(j+k)} \leq H$, for some $H>1$. (moderate growth)

Example 2.2. Given $s \geq 1, \mathcal{G}^{s}=\left\{(n !)^{s-1}\right\}_{n \in \mathbf{N}_{0}}$ is a weight sequence. In fact,

$$
\left[\frac{(n+1) ! \cdot(n-1) !}{(n !)^{2}}\right]^{s-1}=\left[\frac{n+1}{n}\right]^{s-1} \geq 1 \text { and }\left[\frac{(j+k) !}{j ! \cdot k !}\right]^{s-1} \leq\left(2^{s-1}\right)^{j+k} \text {. }
$$

Example 2.3. Let $\sigma>0$ and put $\ell_{n}=[\log (n+e-1)]^{\sigma \cdot n}$. Then

$$
\frac{\ell_{n+1} \cdot \ell_{n-1}}{\ell_{n}^{2}}=\left(\frac{[\log (n+e)]^{(n+1)}}{[\log (n+e-1)]^{n}} \cdot \frac{[\log (n+e-2)]^{(n-1)}}{[\log (n+e-1)]^{n}}\right)^{\sigma}=\left(\frac{f(n)}{f(n-1)}\right)^{\sigma} \text {, }
$$

if we set $f:[1,+\infty) \rightarrow \mathbf{R} ; f(x)=\frac{[\log (x+e)]^{(x+1)}}{[\log (x+e-1)]^{x}}$. By analyzing its derivative, we prove that $f$ is nondecreasing and hence logarithmic convexity holds up. Furthermore, given $j, k \in \mathbf{N}$ we have

$$
\begin{aligned}
\frac{\ell_{j+k}}{\ell_{j} \cdot \ell_{k}} & =\left[\frac{\log (j+k+e-1)}{\log (j+e-1)}\right]^{\sigma j} \cdot\left[\frac{\log (j+k+e-1)}{\log (k+e-1)}\right]^{\sigma k} \\
& \leq\left[1+\frac{k}{j+e-1}\right]^{\sigma j} \cdot\left[1+\frac{j}{k+e-1}\right]^{\sigma k} \leq e^{\sigma \cdot(j+k)}
\end{aligned}
$$


which proves moderate growth.

Example 2.4. Consider $m_{n}=\left[\log \left(\log \left(n+e^{e}-1\right)\right)\right]^{\beta \cdot n}$, with $\beta>0$. Analogously to Example 2.3 we study the derivative of $g:[1,+\infty) \rightarrow \mathbf{R}$;

$$
g(x)=\frac{\left[\log \left(\log \left(x+e^{e}\right)\right)\right]^{(x+1)}}{\left[\log \left(\log \left(x+e^{e}-1\right)\right)\right]^{x}}
$$

to confirm logarithmic convexity. On the other hand,

$$
\begin{aligned}
\frac{m_{j+k}}{m_{j} \cdot m_{k}} & =\left[\frac{\log \left(\log \left(j+k+e^{e}-1\right)\right)}{\log \left(\log \left(j+e^{e}-1\right)\right)}\right]^{\beta j} \cdot\left[\frac{\log \left(\log \left(j+k+e^{e}-1\right)\right)}{\log \left(\log \left(k+e^{e}-1\right)\right)}\right]^{\beta k} \\
& \leq\left[\frac{\log \left(j+k+e^{e}-1\right)}{\log \left(j+e^{e}-1\right)}\right]^{\beta j} \cdot\left[\frac{\log \left(j+k+e^{e}-1\right)}{\log \left(k+e^{e}-1\right)}\right]^{\beta k} \\
& \leq\left[1+\frac{k}{j+e^{e}-1}\right]^{\sigma j} \cdot\left[1+\frac{j}{k+e^{e}-1}\right]^{\sigma k} \leq e^{\sigma \cdot(j+k)}
\end{aligned}
$$

checking moderate growth.

Remark 2.5. Given $\mathscr{L}=\left\{\ell_{k}\right\}_{k \in \mathbf{N}_{0}}$ and $\mathscr{M}=\left\{m_{k}\right\}_{k \in \mathbf{N}_{0}}$ weight sequences, their product given by $\mathscr{K}=\left\{\ell_{k} \cdot m_{k}\right\}_{k \in \mathbf{N}_{0}}$ is also a weight sequence.

Proposition 2.6. Let $\mathscr{M}=\left\{m_{n}\right\}_{n \in \mathbf{N}_{0}}$ be a weight sequence. Then following properties hold:

1. $\mathscr{M}$ is non-decreasing.

2. The sequence given by $\beta_{n}:=\left(m_{n}\right)^{\frac{1}{n}}$, for $n \geq 1$, is non-decreasing.

3. Let $k, n \in \mathbf{N}_{0}$ such that $k \leq n$; then

$$
m_{k} \cdot m_{n-k} \leq m_{n}
$$

4. For any $k \in \mathbf{N}$ there exists $C_{\{k\}}>1$ such that:

$$
\sup _{j \geq 1}\left(\frac{m_{j+k}}{m_{j}}\right)^{\frac{1}{j}} \leq C_{\{k\}}
$$

Proof. Let $\omega_{n}=\log m_{n}$; from the definition of weight sequence, we show by induction that

which implies that

$$
\omega_{j} \leq \frac{j}{j+k} \cdot \omega_{j+k}, \quad \forall k \in \mathbf{N}_{0}, \forall j \in \mathbf{N}
$$

$$
\frac{\omega_{n}}{n} \leq \frac{\omega_{n+1}}{n+1} \quad \text { and } \quad \omega_{j}+\omega_{k} \leq \omega_{j+k},
$$

proving 1, 2 and 3; on the other hand, since

$$
\left(\frac{m_{j+1}}{m_{j}}\right) \leq H^{2 j}, \quad \forall j \in \mathbf{N}_{0}
$$

it follows that

$$
\left(\frac{m_{j+k}}{m_{j}}\right)=\left(\frac{m_{j+1}}{m_{j}}\right) \cdot \ldots \cdot\left(\frac{m_{j+k}}{m_{j+k-1}}\right) \leq H^{2[j+(j+1)+\ldots+(j+k-1)]} \leq H^{2 j \cdot\left(k^{2}+k\right)}, \quad \forall j \geq 1 .
$$

Thus 4 is proved by taking $C_{\{k\}}=H^{2\left(k^{2}+k\right)}$. 
Remark 2.7. By applying Proposition 2.6 for the particular case where $s=2$ in Example 2.2, we obtain for each $k \in \mathbf{N}$ the existence of $B_{\{k\}}$ such that

$$
\left(\frac{(j+k) !}{j !}\right)^{\frac{1}{j}} \leq B_{\{k\}} .
$$

Definition 2.8. Let $\mathscr{M}=\left\{m_{n}\right\}_{n \in \mathbf{N}_{0}}$ be a weight sequence; we say that $f \in$ $C^{\infty}\left(\mathbf{T}^{N}\right)$ is ultradifferentiable of Romieu Class $\mathscr{M}$ if there exist constants $C, h>0$ such that

$$
\left|D^{\alpha} f(x)\right| \leq C \cdot h^{|\alpha|} \cdot m_{|\alpha|} \cdot|\alpha| !, \quad \forall \alpha \in \mathbf{N}_{0}^{N}, \forall x \in \mathbf{T}^{N} .
$$

Definition 2.9. We denote the space of ultradifferentiable functions of Romieu class $\mathscr{M}$ as $\mathcal{E}_{\mathscr{M}}\left(\mathbf{T}^{N}\right) . \mathcal{E}_{\mathscr{M}}\left(\mathbf{T}^{N}\right)$ is called the Denjoy-Carleman class on $\mathbf{T}^{N}$ associated to $\mathscr{M}$.

Remark 2.10. Let us analyze the difference between our sequences and the ones introduced by Komatsu in [K1]. In comparison, we have $M_{n}=m_{n} \cdot n$ !; although initial conditions do not exist in [K1], they are essentially imposed to facilitate computations and are not a real obstruction for the theory.

Proceeding to logarithmic convexity, note that

$$
\frac{M_{n-1} \cdot M_{n+1}}{M_{n}^{2}}=\frac{m_{n+1} \cdot m_{n-1} \cdot(n-1) ! \cdot(n+1) !}{m_{n}^{2} \cdot(n !)^{2}}=\frac{m_{n+1} \cdot m_{n-1}}{m_{n}^{2}} \cdot \frac{n+1}{n} .
$$

Hence condition 2 in Definition 2.1 is slightly stronger than (M.1) established in [K1]. The reason for requiring it becomes evident in Proposition 2.6 and Lemma 5.10.

Finally, Property 3 in Definition 2.1 is named "stability under ultradifferentiable operators" in [K1]. It implies the result stated in Lemma 4.17, which is crucial in the proofs of Theorems 4.18, 5.2, 5.9 and 5.12. In the ultradifferentiable context, it implies the associated function (Remark 4.6) is a weight function (see [BMM], $[\mathrm{BMT}])$, relating two different ways of defining ultradifferentiable functions.

It is not difficult to check that $\mathcal{E}_{\mathscr{M}}\left(\mathbf{T}^{N}\right)$ is a vector subspace of $C^{\infty}\left(\mathbf{T}^{N}\right)$. Moreover, each statement proved in Proposition 2.6 has a direct consequence on the classes associated. The fact that a weight sequence is non-decreasing (1) means that $C^{\omega}\left(\mathbf{T}^{N}\right) \subset \mathcal{E}_{\mathscr{M}}\left(\mathbf{T}^{N}\right)$. It follows from 2 that $\mathcal{E}_{\mathscr{M}}\left(\mathbf{T}^{N}\right)$ is always closed for composition and inverse-closed (see [BM], [RudI]). As implications of 3 and 4 , it is possible to show that any class is a subalgebra of $\mathcal{E}\left(\mathbf{T}^{N}\right)$ and closed by differentiation respectively.

Another important subject related to those spaces of ultradifferentiable functions, the one which actually originated its study, is the existence of elements which are flat at some point.

Definition 2.11. Let $\mathscr{M}$ be a weight sequence; we say that $\mathcal{E}_{\mathscr{M}}\left(\mathbf{T}^{N}\right)$ is quasianalytic if

$$
f \in \mathcal{E}_{\mathscr{M}}\left(\mathbf{T}^{N}\right), x_{0} \in \mathbf{T}^{N} \mid D^{\alpha} f\left(x_{0}\right)=0, \forall \alpha \in \mathbf{N}_{0}^{N} \Rightarrow f \equiv 0 .
$$

The characterization for quasianalyticity was first given partially by Denjoy ([Den]) and then completely by Carleman ([Car]). For more details, we recommend [Hor], [RudII].

Theorem 2.12. $\mathcal{E}_{\mathscr{M}}\left(\mathbf{T}^{N}\right)$ is quasianalytic if and only if

$$
\sum_{j=0}^{+\infty} \frac{m_{j}}{m_{j+1} \cdot(j+1)}=+\infty \text {. }
$$


We now go back to the examples given previously. The classes associated to the sequences set in Example 2.2 are the famous Gevrey classes, which are denoted as $\mathcal{G}^{s}\left(\mathbf{T}^{N}\right)$. When $s=1$, we obtain the class of real analytic functions, which is quasianalytic. If $s>1$, they are non-quasianalytic.

Example 2.3 is very important for comprehension of the gap between smooth functions and real analytic functions, since the particular case where $\sigma=1$ represents the intersection of all inverse-closed non-quasianalytic classes [RudI]. Besides, it is quasianalytic for $0<\sigma \leq 1$ and non-quasianalytic for $1<\sigma$ [Th]. Example 2.4 is historically relevant, since the case where $\beta=1$ was introduced by Denjoy in [Den] as the first example of class of quasianalytic functions which are nowhere analytic.

Before the end of this section, we discuss about inclusion of classes and how this subject is related to the asymptotic behavior of their associated sequences.

Definition 2.13. Let $\mathscr{L}, \mathscr{M}$ be weight sequences. We denote

$$
\mathscr{L} \preceq \mathscr{M} \Leftrightarrow \sup _{k \in \mathbf{N}_{0}}\left(\frac{\ell_{k}}{m_{k}}\right)^{\frac{1}{k}}<+\infty .
$$

Besides, we write $\mathscr{L} \approx \mathscr{M}$ when $\mathscr{L} \preceq \mathscr{M}$ and $\mathscr{M} \preceq \mathscr{L}$.

It is possible to prove (see [Th]) that

$$
\mathcal{E}_{\mathscr{L}}\left(\mathbf{T}^{N}\right) \subset \mathcal{E}_{\mathscr{M}}\left(\mathbf{T}^{N}\right) \Leftrightarrow \mathscr{L} \preceq \mathscr{M}
$$

In particular, $\mathcal{E}_{\mathscr{L}}\left(\mathbf{T}^{N}\right)=\mathcal{E}_{\mathscr{M}}\left(\mathbf{T}^{N}\right)$ if and only if $\mathscr{L} \approx \mathscr{M}$. This shows for instance that $r<s$ implies $\mathcal{G}^{r}\left(\mathbf{T}^{N}\right) \subsetneq \mathcal{G}^{s}\left(\mathbf{T}^{N}\right)$ and $C^{\omega}\left(\mathbf{T}^{N}\right)=\mathcal{E}_{\mathscr{M}}\left(\mathbf{T}^{N}\right) \Leftrightarrow \sup _{j \geq 1}\left(m_{j}\right)^{\frac{1}{j}}<+\infty$.

\section{The topology of $\mathcal{E}_{\mathscr{M}}\left(\mathrm{T}^{N}\right)$ and its dual space}

From now on $\mathscr{M}$ represents an arbitrary weight sequence fixed, since all the results stated only rely on conditions established in Definition 2.1.

Definition 3.1. For any $h>0$, we set

$$
\mathcal{E}_{\mathscr{M}, h}\left(\mathbf{T}^{N}\right)=\left\{f \in \mathcal{E}_{\mathscr{M}}\left(\mathbf{T}^{N}\right) ; \sup _{\alpha \in \mathbf{N}_{0}^{N}}\left[\frac{\left\|D^{\alpha} f\right\|_{\infty}}{h^{|\alpha|} \cdot m_{|\alpha|} \cdot|\alpha| !}\right]<\infty\right\} .
$$

Moreover, we denote for $f \in \mathcal{E}_{\mathscr{M}, h}\left(\mathbf{T}^{N}\right)$,

$$
\|f\|_{\mathscr{M}, h}:=\sup _{\alpha \in \mathbf{N}_{0}^{N}}\left[\frac{\left\|D^{\alpha} f\right\|_{\infty}}{h^{|\alpha|} \cdot m_{|\alpha|} \cdot|\alpha| !}\right] .
$$

If $\mathcal{E}_{\mathscr{M}, h}\left(\mathbf{T}^{N}\right)$ is equipped with $\|.\|_{\mathscr{M}, h}$, which in that case is a norm, it becomes a Banach space.

Proposition 3.2. Let $h_{1}, h_{2} \in \mathbf{R}_{+}$, with $h_{1}<h_{2}$; then $\mathcal{E}_{\mathscr{M}, h_{1}}\left(\mathbf{T}^{N}\right) \subset \mathcal{E}_{\mathscr{M}, h_{2}}\left(\mathbf{T}^{N}\right)$ and the inclusion $\mathcal{E}_{\mathscr{M}, h_{1}}\left(\mathbf{T}^{N}\right) \hookrightarrow \mathcal{E}_{\mathscr{M}, h_{2}}\left(\mathbf{T}^{N}\right)$ is compact.

Proof. Since the inclusion is trivial, we only prove the compactness: let $\left\{f_{n}\right\}_{n \in \mathbf{N}}$ be a bounded sequence in $\mathcal{E}_{\mathscr{M}, h_{1}}\left(\mathbf{T}^{N}\right)$. Thus there exists $C_{0}>0$ such that

$$
\left|D^{\alpha} f_{n}(x)\right| \leq C_{0} \cdot h_{1}^{|\alpha|} \cdot m_{|\alpha|} \cdot|\alpha| !, \quad \forall x \in \mathbf{T}^{N}, \forall \alpha \in \mathbf{N}_{0}^{N}, \quad \forall n \in \mathbf{N},
$$

and consequently for every $k \in \mathbf{N}_{0}$ we are able to find $C_{k}>0$ such that

$$
\sum_{|\beta| \leq k} \sup _{x \in \mathbf{T}^{N}}\left|D^{\beta} f_{n}(x)\right| \leq C_{k}, \forall n \in \mathbf{N} \text {. }
$$


By Arzelà-Ascoli Theorem there exists a subsequence $\left\{f_{n_{k}}\right\}_{k \in \mathbf{N}}$ converging to $f$ in $C^{\infty}\left(\mathbf{T}^{N}\right)$. Besides, for any $x \in \mathbf{T}^{N}, \gamma \in \mathbf{N}_{0}^{N}$,

$$
\left|D^{\gamma} f(x)\right|=\lim _{k \rightarrow+\infty}\left|D^{\gamma} f_{n_{k}}(x)\right| \leq C_{0} \cdot h_{2}^{|\gamma|} \cdot m_{|\gamma|} \cdot|\gamma| !,
$$

which allows us to conclude that $f \in \mathcal{E}_{\mathscr{M}, h_{2}}\left(\mathbf{T}^{N}\right)$. It remains to prove that $f_{n_{k}} \rightarrow f$ in $\mathcal{E}_{\mathscr{M}, h_{2}}\left(\mathbf{T}^{N}\right)$.

Given $\varepsilon>0$, we take $p \in \mathbf{N}$ such that $\left(\frac{h_{1}}{h_{2}}\right)^{p} \leq \frac{\varepsilon}{2 C_{0}}$ and

$$
C_{1}:=\max \left\{\frac{1}{h_{2}^{q} \cdot m_{q} \cdot q !} ; 0 \leq q \leq p\right\} .
$$

Due to the fact that $f_{n_{k}} \rightarrow f$ in $C^{\infty}\left(\mathbf{T}^{N}\right)$, it is possible to find $k_{1} \in \mathbf{N}$ such that

$$
\sup _{x \in \mathbf{T}^{N}}\left|D^{\lambda} f_{n_{k}}(x)-D^{\lambda} f(x)\right| \leq \frac{\varepsilon}{C_{1}}, \quad|\lambda| \leq p, \quad k \geq k_{1} .
$$

Let $k \geq k_{1} ;$ if $|\lambda| \leq p$

$$
\sup _{x \in \mathbf{T}^{N}}\left[\frac{\left|D^{\lambda} f_{n_{k}}(x)-D^{\lambda} f(x)\right|}{h_{2}^{|\lambda|} \cdot m_{|\lambda|} \cdot|\lambda| !}\right] \leq \frac{\varepsilon}{C_{1}} \cdot C_{1}=\varepsilon .
$$

When $|\lambda|>p$,

$$
\begin{aligned}
\sup _{x \in \mathbf{T}^{N}}\left[\frac{\left|D^{\lambda} f_{n_{k}}(x)-D^{\lambda} f(x)\right|}{h_{2}^{|\lambda|} \cdot m_{|\lambda|} \cdot|\lambda| !}\right] & \leq \sup _{x \in \mathbf{T}^{N}}\left[\frac{\left|D^{\lambda} f_{n_{k}}(x)-D^{\lambda} f(x)\right|}{h_{1}^{|\lambda|} \cdot m_{|\lambda|} \cdot|\lambda| !}\right] \cdot\left(\frac{h_{1}}{h_{2}}\right)^{p} \\
& \leq\left[\left\|f_{n_{k}}\right\|_{\mathscr{M}, h_{1}}+\|f\|_{\mathscr{M}, h_{1}}\right] \cdot \frac{\varepsilon}{2 C_{0}} \leq \varepsilon
\end{aligned}
$$

Therefore $\left\|f_{n_{k}}-f\right\|_{\mathscr{M}, h_{2}} \leq \varepsilon$ for all $k \geq k_{1}$, which shows that $f_{n_{k}} \rightarrow f$ in $\mathcal{E}_{\mathscr{M}, h_{2}}\left(\mathbf{T}^{N}\right)$.

Let $\left\{h_{n}\right\}_{n \in \mathbf{N}}$ be a strictly increasing sequence of positive real numbers such that $h_{n} \rightarrow+\infty$. It is not difficult to see that

$$
\mathcal{E}_{\mathscr{M}}\left(\mathbf{T}^{N}\right)=\bigcup_{n \in \mathbf{N}} \mathcal{E}_{\mathscr{M}, h_{n}}\left(\mathbf{T}^{N}\right)
$$

We endow $\mathcal{E}_{\mathscr{M}}\left(\mathbf{T}^{N}\right)$ with the inductive limit topology given by the family of $\mathcal{E}_{\mathscr{M}, h_{n}}\left(\mathbf{T}^{N}\right)$. That is,

$$
\mathcal{E}_{\mathscr{M}}\left(\mathbf{T}^{N}\right)=\underset{n \in \mathbf{N}}{\lim _{\vec{\in}}} \mathcal{E}_{\mathscr{M}, h_{n}}\left(\mathbf{T}^{N}\right)
$$

It is possible to prove that such topology does not depend on the choice of $\left\{h_{n}\right\}_{n \in \mathbf{N}}$. Moreover, it follows from Proposition 3.2 that $\mathcal{E}_{\mathscr{M}}\left(\mathbf{T}^{N}\right)$ is an injective limit of a compact sequence of locally convex spaces, also known in the literature as a DFS space. For more details, we recommend [K2].

Now that we have established a topology for $\mathcal{E}_{\mathscr{M}}\left(\mathbf{T}^{N}\right)$, it is possible introduce the space of ultradistributions, which is absolutely fundamental for the theory of linear partial differential equations.

Definition 3.3. We define $\mathscr{D}_{\mathscr{M}}^{\prime}\left(\mathbf{T}^{N}\right)$ as the topological dual space of $\mathcal{E}_{\mathscr{M}}\left(\mathbf{T}^{N}\right)$. That is, the space of continuous linear functionals $u: \mathcal{E}_{\mathscr{M}}\left(\mathbf{T}^{N}\right) \rightarrow \mathbf{C}$.

Theorem 3.4. Let $u: \mathcal{E}_{\mathscr{M}}\left(\mathbf{T}^{N}\right) \rightarrow \mathbf{C}$ be a linear functional; the following conditions are equivalent: 
1. $u \in \mathscr{D}_{\mathscr{M}}^{\prime}\left(\mathbf{T}^{N}\right)$.

2. For every $\varepsilon>0$, there exists $C_{\varepsilon}>0$ such that

$$
|\langle u, \varphi\rangle| \leq C_{\varepsilon} \sup _{\alpha \in \mathbf{N}_{0}^{N}}\left(\frac{\left\|\partial^{\alpha} \varphi\right\|_{\infty} \cdot \varepsilon^{|\alpha|}}{m_{|\alpha|} \cdot|\alpha| !}\right), \quad \forall \varphi \in \mathcal{E}_{\mathscr{M}}\left(\mathbf{T}^{N}\right) .
$$

3. If $\left\{\varphi_{n}\right\}_{n \in \mathbf{N}} \subset \mathcal{E}_{\mathscr{M}}\left(\mathbf{T}^{N}\right)$ converges to 0 in $\mathcal{E}_{\mathscr{M}}\left(\mathbf{T}^{N}\right)$, then $\left\langle u, \varphi_{n}\right\rangle \rightarrow 0$.

Proof. $1 \Rightarrow 2$ : If 2 does not hold, there exist $\varepsilon_{0}>0$ and $\left\{\varphi_{n}\right\}_{n \in \mathbf{N}} \subset \mathcal{E}_{\mathscr{M}}\left(\mathbf{T}^{N}\right)$ such that

$$
\left|\left\langle u, \varphi_{n}\right\rangle\right|>n \cdot \sup _{\alpha \in \mathbf{N}_{0}^{N}}\left(\frac{\left\|\partial^{\alpha} \varphi_{n}\right\|_{\infty} \cdot \varepsilon_{0}^{|\alpha|}}{m_{|\alpha|} \cdot|\alpha| !}\right) .
$$

By putting $\psi_{n}=\frac{\varphi_{n}}{\left|\left\langle u, \varphi_{n}\right\rangle\right|}$, we get that

$$
1>n \cdot \sup _{\alpha \in \mathbf{N}_{0}^{N}}\left(\frac{\left\|\partial^{\alpha} \psi_{n}\right\|_{\infty} \cdot \varepsilon_{0}^{|\alpha|}}{m_{|\alpha|} \cdot|\alpha| !}\right) \Rightarrow \sup _{\alpha \in \mathbf{N}_{0}^{N}}\left(\frac{\left\|\partial^{\alpha} \psi_{n}\right\|_{\infty} \cdot \varepsilon_{0}^{|\alpha|}}{m_{|\alpha|} \cdot|\alpha| !}\right)<\frac{1}{n},
$$

which implies that

$$
\left|\partial^{\alpha} \psi_{n}(x)\right| \leq\left(\frac{1}{\varepsilon_{0}}\right)^{|\alpha|} \cdot m_{|\alpha|} \cdot|\alpha| !, \quad \forall x \in \mathbf{T}^{N}, \quad \forall \alpha \in \mathbf{N}_{0}^{N} .
$$

Therefore $\left\{\psi_{n}\right\}_{n \in \mathbf{N}} \subset \mathcal{E}_{\mathscr{M}, 1 / \varepsilon_{0}}\left(\mathbf{T}^{N}\right)$ and

$$
\left|\left\langle u, \psi_{n}\right\rangle\right| \geq n\left\|\psi_{n}\right\|_{\mathscr{M}, 1 / \varepsilon_{0}}, \quad \forall n \in \mathbf{N},
$$

which shows that $\left.u\right|_{\mathcal{E}_{\mathscr{M}, 1 / \varepsilon_{0}}\left(\mathbf{T}^{N}\right)}$ is not continuous and hence $u$ is not continuous.

$2 \Rightarrow 3$ : Let $\left\{\varphi_{n}\right\}_{n \in \mathbf{N}} \subset \mathcal{E}_{\mathscr{M}}\left(\mathbf{T}^{N}\right)$ a sequence that converges to 0 . By a property of DFS spaces, there exists $h>0$ such that $\left(\varphi_{n}\right)_{n \in \mathbf{N}} \subset \mathcal{E}_{\mathscr{M}, h}\left(\mathbf{T}^{N}\right)$ and $\left(\varphi_{n}\right)_{n \in \mathbf{N}} \rightarrow 0$ in $\mathcal{E}_{\mathscr{M}, h}\left(\mathbf{T}^{N}\right)$. If we set $\varepsilon=\frac{1}{h}$, one can find $C>0$ satisfying

$$
\left|\left\langle u, \varphi_{n}\right\rangle\right| \leq C \cdot \sup _{\alpha \in \mathbf{N}_{0}^{N}}\left(\frac{\left\|\partial^{\alpha} \varphi_{n}\right\|_{\infty}}{h^{|\alpha|} \cdot m_{|\alpha|} \cdot|\alpha| !}\right) \leq C \cdot\left\|\varphi_{n}\right\|_{\mathscr{M}, h} .
$$

Since $\left\|\varphi_{n}\right\|_{\mathscr{M}, h} \rightarrow 0$, it follows that $\left|\left\langle u, \varphi_{n}\right\rangle\right| \rightarrow 0$.

$3 \Rightarrow 1$ : If $u \notin \mathscr{D}_{\mathscr{M}}^{\prime}\left(\mathbf{T}^{N}\right)$, there exists $h>0$ such that $\left.u\right|_{\mathcal{E}_{\mathscr{M}, h}\left(\mathbf{T}^{N}\right)}$ is not continuous. Thus for every $j \in \mathbf{N}$ we may obtain $\varphi_{j} \in \mathcal{E}_{\mathscr{M}, h}\left(\mathbf{T}^{N}\right)$ such that $\left|\left\langle u, \varphi_{j}\right\rangle\right|>j\left\|\varphi_{j}\right\|_{\mathscr{M}, h}$. Let $\psi_{j}:=\frac{\varphi_{j}}{\left|\left\langle u, \varphi_{j}\right\rangle\right|} ;$ then

$$
\left|\left\langle u, \psi_{j}\right\rangle\right|=1 \quad \text { and } \quad\left\|\psi_{j}\right\|_{\mathscr{M}, h}=\frac{\left\|\varphi_{j}\right\|_{\mathscr{M}, h}}{\left|\left\langle u, \varphi_{j}\right\rangle\right|}<\frac{1}{j}, \quad \forall j \in \mathbf{N} .
$$

Thus $\left\{\psi_{j}\right\}_{j \in \mathbf{N}} \rightarrow 0$ in $\mathcal{E}_{\mathscr{M}}\left(\mathbf{T}^{N}\right)$ whereas $\left\langle u, \psi_{j}\right\rangle \not \rightarrow 0$, which proves our claim by contradiction.

The notion of convergence here, analogously to other spaces of distributions, is the pointwise convergence. That is,

Definition 3.5. Let $\left\{u_{n}\right\}_{n \in \mathbf{N}}$ be a sequence in $\mathscr{D}_{\mathscr{M}}^{\prime}\left(\mathbf{T}^{N}\right)$ and $u \in \mathscr{D}_{\mathscr{M}}^{\prime}\left(\mathbf{T}^{N}\right)$. We say that $u_{n} \rightarrow u$ if

$$
\left\langle u_{n}, \varphi\right\rangle \rightarrow\langle u, \varphi\rangle, \quad \forall \varphi \in \mathcal{E}_{\mathscr{M}}\left(\mathbf{T}^{N}\right) .
$$

We also have the following completeness condition: 
Lemma 3.6. Let $\left\{u_{n}\right\}_{n \in \mathbf{N}}$ be a sequence in $\mathscr{D}_{\mathscr{M}}^{\prime}\left(\mathbf{T}^{N}\right)$ such that $\left\langle u_{n}, \varphi\right\rangle$ is a Cauchy sequence for every $\varphi \in \mathcal{E}_{\mathscr{M}}\left(\mathbf{T}^{N}\right)$. Then there exists $u \in \mathscr{D}_{\mathscr{M}}^{\prime}\left(\mathbf{T}^{N}\right)$ such that

$$
\lim \left\langle u_{n}, \varphi\right\rangle=\langle u, \varphi\rangle, \quad \forall \varphi \in \mathcal{E}_{\mathscr{M}}\left(\mathbf{T}^{N}\right) .
$$

Proof. Set $u: \mathcal{E}_{\mathscr{M}}\left(\mathbf{T}^{N}\right) \rightarrow \mathbf{C} ;\langle u, \varphi\rangle=\lim \left\langle u_{n}, \varphi\right\rangle$. We claim $u$ is continuous; in fact, let $\left\{\varphi_{k}\right\}_{k \in \mathbf{N}}$ be a sequence in $\mathcal{E}_{\mathscr{M}}\left(\mathbf{T}^{N}\right)$ converging to 0 . Then there exists $h>0$ such that $\varphi_{k} \in \mathcal{E}_{\mathscr{M}, h}\left(\mathbf{T}^{N}\right)$ for every $k \in \mathbf{N}$ and $\left\|\varphi_{k}\right\|_{\mathscr{M}, h} \rightarrow 0$.

For any $\psi \in \mathcal{E}_{\mathscr{M}, h}\left(\mathbf{T}^{N}\right)$, sequence $\left\langle u_{n}, \psi\right\rangle$ is bounded. Since $\left.u_{n}\right|_{\mathcal{E}_{\mathscr{M}, h}\left(\mathbf{T}^{N}\right)}$ is continuous for each $n$, it follows from Uniform Boundedness Principle the existence of $M>0$ such that

$$
\left|\left\langle u_{n}, \psi\right\rangle\right| \leq M \cdot\|\psi\|_{\mathscr{M}, h}, \quad \forall n \in \mathbf{N}, \forall \psi \in \mathcal{E}_{\mathscr{M}, h}\left(\mathbf{T}^{N}\right) .
$$

Given $\varepsilon>0$, we choose $k_{0} \in \mathbf{N}$ such that

$$
\left|\left\langle u_{n}, \varphi_{k}\right\rangle\right| \leq \frac{\varepsilon}{2}, \quad \forall n \in \mathbf{N}, \forall k \geq k_{0} .
$$

Because $\left\langle u, \varphi_{k}\right\rangle=\lim _{n}\left\langle u_{n}, \varphi_{k}\right\rangle$, for each $k \geq k_{0}$ we are able to find $n_{k} \in \mathbf{N}$ satisfying

$$
\left|\left\langle u, \varphi_{k}\right\rangle-\left\langle u_{n_{k}}, \varphi_{k}\right\rangle\right| \leq \frac{\varepsilon}{2}
$$

Therefore for every $k \geq k_{0}$, we have

$$
\left|\left\langle u, \varphi_{k}\right\rangle\right| \leq\left|\left\langle u, \varphi_{k}\right\rangle-\left\langle u_{n_{k}}, \varphi_{k}\right\rangle\right|+\left|\left\langle u_{n_{k}}, \varphi_{k}\right\rangle\right| \leq \varepsilon,
$$

which proves our assertion, by Theorem 3.4.

\section{Fourier series for $\mathcal{E}_{\mathscr{M}}\left(\mathrm{T}^{N}\right)$ and $\mathscr{D}_{\mathscr{M}}^{\prime}\left(\mathrm{T}^{N}\right)$}

4.1. Fourier series. We begin the subsection setting Fourier coefficients for elements of $\mathcal{E}_{\mathscr{M}}\left(\mathbf{T}^{N}\right)$ and showing that any $\varphi \in \mathcal{E}_{\mathscr{M}}\left(\mathbf{T}^{N}\right)$ is completely described by its Fourier Series.

Definition 4.1. Let $\varphi \in \mathcal{E}_{\mathscr{M}}\left(\mathbf{T}^{N}\right)$; for each $\xi \in \mathbf{Z}^{N}$ we define its Fourier coefficient as:

$$
\hat{\varphi}(\xi):=\frac{1}{(2 \pi)^{N}} \int_{\mathbf{T}^{N}} e^{-i x \xi} \varphi(x) d x .
$$

Theorem 4.2. For every $\varphi \in \mathcal{E}_{\mathscr{M}}\left(\mathbf{T}^{N}\right)$ we have

$$
\varphi(x)=\sum_{\xi \in \mathbf{Z}^{N}} \hat{\varphi}(\xi) \cdot e^{i x \xi}, \quad \forall x \in \mathbf{T}^{N},
$$

with convergence in $\mathcal{E}_{\mathscr{M}}\left(\mathbf{T}^{N}\right)$. Furthermore, there exist constants $C, \delta>0$ such that

$$
|\hat{\varphi}(\xi)| \leq C \cdot \inf _{n \in \mathbf{N}_{0}}\left(\frac{m_{n} \cdot n !}{\delta^{n} \cdot(1+|\xi|)^{n}}\right), \quad \forall \xi \in \mathbf{Z}^{N}
$$

Proof. We start by proving (4.1); when $\xi=0$ the estimate is obvious for $\delta=1$, so we may consider $\xi \neq 0$. It is easy to see that $|\hat{\varphi}(\xi)| \leq\|\varphi\|_{\infty}$; for any non-zero $\alpha \in \mathbf{N}_{0}^{N}, \widehat{D^{\alpha} \varphi}(\xi)=\xi^{\alpha} \cdot \widehat{\varphi}(\xi)$. Hence there exist $C_{1}, h_{1}>1$ such that

$$
\left|\xi^{\alpha}\right| \cdot|\hat{\varphi}(\xi)| \leq C_{1} \cdot h_{1}^{|\alpha|} \cdot m_{|\alpha|} \cdot|\alpha| !
$$


Since $|\xi|^{|\alpha|} \leq \sum_{|\beta|=|\alpha|} \frac{|\alpha| !}{\beta !} \cdot\left|\xi^{\beta}\right|$, it follows that

$$
\begin{aligned}
|\xi|^{|\alpha|} \cdot|\hat{\varphi}(\xi)| & \leq C_{1} \cdot h_{1}^{|\alpha|} \cdot m_{|\alpha|} \cdot|\alpha| ! \cdot \sum_{|\beta|=|\alpha|} \frac{|\alpha| !}{\beta !} \\
& \leq C_{1} \cdot\left(h_{1} \cdot N\right)^{|\alpha|} \cdot m_{|\alpha|} \cdot|\alpha| ! .
\end{aligned}
$$

Because $\xi \neq 0$, we infer that

$$
(1+|\xi|)^{|\alpha|} \cdot|\hat{\varphi}(\xi)| \leq C_{1} \cdot\left(2 \cdot h_{1} \cdot N\right)^{|\alpha|} \cdot m_{|\alpha|} \cdot|\alpha| !,
$$

which proves the estimate if we take $C=\max \left\{\|\varphi\|_{\infty}, C_{1}\right\}$ and $\delta=\frac{1}{2 \cdot h_{1} \cdot N}$.

Proceeding to the proof of the first statement, note that since $\varphi$ is smooth, the convergence holds in $C^{\infty}\left(\mathbf{T}^{N}\right)$. For each $k \in \mathbf{N}$, consider the partial sum

$$
S_{k} \varphi(x)=\sum_{|\xi| \leq k} \hat{\varphi}(\xi) \cdot e^{i \xi x} .
$$

Observe that $S_{k}$ is real analytic and consequently an element of $\mathcal{E}_{\mathscr{M}}\left(\mathbf{T}^{N}\right)$. Besides, for any $\alpha \in \mathbf{N}_{0}^{N}$

$$
D^{\alpha}\left(\varphi-S_{k} \varphi\right)(x)=\sum_{|\xi| \geq k+1} \hat{\varphi}(\xi) \cdot \xi^{\alpha} \cdot e^{i \xi x} .
$$

Thereafter it follows from (4.1) that for any $x \in \mathbf{T}^{N}, \alpha \in \mathbf{N}_{0}^{N}$,

$$
\begin{aligned}
\left|D^{\alpha}\left(\varphi-S_{k} \varphi\right)(x)\right| & \leq C\left(\frac{1}{\delta}\right)^{|\alpha|+2 N} m_{|\alpha|+2 N} \cdot(|\alpha|+2 N) ! \sum_{|\xi| \geq k+1}(1+|\xi|)^{-2 N} \\
& \leq C\left(\frac{1}{\delta}\right)^{|\alpha|+2 N}\left(m_{|\alpha|} \cdot C_{\{2 N\}}^{|\alpha|}\right) \cdot\left(|\alpha| ! \cdot B_{\{2 N\}}^{|\alpha|}\right) \sum_{|\xi| \geq k+1}(1+|\xi|)^{-2 N} \\
& \leq C^{\prime} \cdot h_{1}^{\prime|\alpha|} \cdot m_{|\alpha|} \cdot|\alpha| !
\end{aligned}
$$

by applying (2.1), (2.2) and setting

$$
C^{\prime}=C\left(\frac{1}{\delta}\right)^{2 N} \sum_{\xi \in \mathbf{Z}^{N}}(1+|\xi|)^{-2 N}, \quad h_{1}^{\prime}=\left(\frac{1}{\delta}\right) B_{\{2 N\}} C_{\{2 N\}} .
$$

Since $h_{1}<h_{1}^{\prime}$ and $\varphi,\left(\varphi-S_{k} \varphi\right)$ belong to $\mathcal{E}_{\mathscr{M}, h_{1}^{\prime}}\left(\mathbf{T}^{N}\right)$, the same holds for every $S_{k} \varphi$. In addition,

$\left|D^{\alpha}\left(\varphi-S_{k} \varphi\right)(x)\right| \leq\left[\left(\frac{C}{\delta}\right)^{2 N} \sum_{|\xi| \geq k+1}(1+|\xi|)^{-2 N}\right]\left(h_{1}^{\prime}\right)^{|\alpha|} \cdot m_{|\alpha|} \cdot|\alpha| !, \forall x \in \mathbf{T}^{N}, \forall \alpha \in \mathbf{N}_{0}^{N}$.

Thus $\lim _{k \rightarrow+\infty}\left\|\varphi-S_{k} \varphi\right\|_{\mathscr{M}, h_{1}^{\prime}}=0$ and $S_{k} \varphi \rightarrow \varphi$ in $\mathcal{E}_{\mathscr{M}}\left(\mathbf{T}^{N}\right)$.

We now prove the converse of Theorem 4.2:

Theorem 4.3. Let $\left\{b_{\xi}\right\}_{\xi \in \mathbf{Z}^{N}}$ be a sequence in $\mathbf{C}$ and assume the existence of $C, \delta>0$ satisfying

$$
\left|b_{\xi}\right| \leq C \cdot \inf _{n \in \mathbf{N}_{0}}\left(\frac{m_{n} \cdot n !}{\delta^{n} \cdot(1+|\xi|)^{n}}\right), \quad \forall \xi \in \mathbf{Z}^{N} .
$$


Then there exists $\psi \in \mathcal{E}_{\mathscr{M}}\left(\mathbf{T}^{N}\right)$ such that

$$
\psi(x)=\sum_{\xi \in \mathbf{Z}^{N}} b_{\xi} \cdot e^{i x \xi}
$$

with convergence in $\mathcal{E}_{\mathscr{M}}\left(\mathbf{T}^{N}\right)$. In addition, $\widehat{\psi}(\xi)=b_{\xi}$.

Proof. Let $\psi: \mathbf{T}^{N} \rightarrow \mathbf{C}$ given by $\psi(x)=\sum_{\xi \in \mathbf{Z}^{N}} b_{\xi} \cdot e^{i x \xi} ;$ it follows from hypothesis that $\psi$ is smooth, with its sequence of partial sums converging to $\psi$ in $C^{\infty}\left(\mathbf{T}^{N}\right)$. For any $\alpha \in \mathbf{N}_{0}^{N}$,

$$
\begin{aligned}
\left|D^{\alpha} \psi(x)\right| & \leq \sum_{\xi \in \mathbf{Z}^{N}}(1+|\xi|)^{|\alpha|} \cdot\left|b_{\xi}\right| \\
& \leq \sum_{\xi \in \mathbf{Z}^{N}}\left(C \cdot \frac{m_{|\alpha|+2 N} \cdot(|\alpha|+2 N) !}{\delta^{|\alpha|+2 N}}\right) \cdot \frac{1}{(1+|\xi|)^{2 N}} \\
& \leq\left(C\left(\frac{1}{\delta}\right)^{2 N} \sum_{\xi \in \mathbf{Z}^{N}} \frac{1}{(1+|\xi|)^{2 N}}\right)\left(\frac{C_{\{2 N\}} \cdot B_{\{2 N\}}}{\delta}\right)^{|\alpha|} \cdot m_{|\alpha|} \cdot|\alpha| !,
\end{aligned}
$$

which shows us that $\psi \in \mathcal{E}_{\mathscr{M}}\left(\mathbf{T}^{N}\right)$. By setting $S_{j} \psi(x)=\sum_{|\xi| \leq j} b_{\xi} \cdot e^{i x \xi}$ for each $j \in \mathbf{N}$, we obtain

$$
\left|D^{\alpha}\left(\psi-S_{j} \psi\right)(x)\right| \leq C \cdot\left(\frac{1}{\delta}\right)^{2 N} \sum_{|\xi| \geq j+1} \frac{1}{(1+|\xi|)^{2 N}}\left(\frac{C_{\{2 N\}} \cdot B_{\{2 N\}}}{\delta}\right)^{|\alpha|} \cdot m_{|\alpha|} \cdot|\alpha| ! .
$$

So $\lim _{j \rightarrow \infty}\left\|\psi-S_{j} \psi\right\|_{\mathscr{M}, h_{1}}=0$ for $h_{1}=\frac{C_{\{2 N\}} \cdot B_{\{2 N\}}}{\delta}$, which implies that $S_{j} \psi \rightarrow \psi$ in $\mathcal{E}_{\mathscr{M}}\left(\mathbf{T}^{N}\right)$.

Next we extend the concept of Fourier coefficient and prove versions of Theorems 4.2 and 4.3 for ultradistributions.

Definition 4.4. Let $u \in \mathscr{D}_{\mathscr{M}}^{\prime}\left(\mathbf{T}^{N}\right)$; we define its Fourier Coefficient $\hat{u}(\xi)$ as

$$
\hat{u}(\xi)=\frac{1}{(2 \pi)^{N}} \cdot\left\langle u, e^{-i x \xi}\right\rangle, \quad \forall \xi \in \mathbf{Z}^{\mathbf{N}} .
$$

Theorem 4.5. Let $u \in \mathscr{D}_{\mathscr{M}}^{\prime}\left(\mathbf{T}^{N}\right)$; then for every $\varepsilon>0$, there exists $C_{\varepsilon}>0$ such that

$$
|\hat{u}(\xi)| \leq C_{\varepsilon} \sup _{n \in \mathbf{N}_{0}}\left(\frac{\varepsilon^{n} \cdot(1+|\xi|)^{n}}{m_{n} \cdot n !}\right), \quad \forall \xi \in \mathbf{Z}^{N} .
$$

Proof. For every $\varepsilon>0, \xi \in \mathbf{Z}^{N}$ it follows from (3.1) the existence of $C_{\varepsilon}>0$ satisfying

$$
|\hat{u}(\xi)| \leq C_{\varepsilon} \sup _{\substack{x \in \mathbf{T}^{N} \\ \alpha \in \mathbf{N}_{0}^{N}}}\left(\frac{\left|\partial_{x}^{\alpha}\left(e^{-i x \xi}\right)\right| \cdot \varepsilon^{|\alpha|}}{m_{|\alpha|} \cdot|\alpha| !}\right)
$$


which implies that

$$
\begin{aligned}
|\hat{u}(\xi)| & \leq C_{\varepsilon} \sup _{\alpha \in \mathbf{N}_{0}^{N}}\left(\frac{\left(1+\left|\xi^{\alpha}\right|\right) \cdot \varepsilon^{|\alpha|}}{m_{|\alpha|} \cdot|\alpha| !}\right) \leq C_{\varepsilon} \sup _{\alpha \in \mathbf{N}_{0}^{N}}\left(\frac{(1+|\xi|)^{|\alpha|} \cdot \varepsilon^{|\alpha|}}{m_{|\alpha|} \cdot|\alpha| !}\right) \\
& =C_{\varepsilon} \sup _{n \in \mathbf{N}_{0}}\left(\frac{\varepsilon^{n} \cdot(1+|\xi|)^{n}}{m_{n} \cdot n !}\right),
\end{aligned}
$$

as we intended to prove.

Remark 4.6. Note that the functions $\inf _{n \in \mathbf{N}_{0}}\left(\frac{m_{n} \cdot n !}{t^{n}}\right)$ and $\sup _{n \in \mathbf{N}_{0}}\left(\frac{t^{n}}{m_{n} \cdot n !}\right)$ naturally show up in Theorems 4.2, 4.3 and 4.5. There is actually a strong connection between them.

In fact, for any $t>0$ we have $\lim _{n \rightarrow+\infty}\left(\frac{t^{n}}{m_{n} \cdot n !}\right)=0$. Since $\sup _{n \in \mathbf{N}_{0}}\left(\frac{t^{n}}{m_{n} \cdot n !}\right) \geq 1$, it follows that $\sup _{n \in \mathbf{N}_{0}}\left(\frac{t^{n}}{m_{n} \cdot n !}\right)$ is always assumed by some $n_{0}$ and a similar argument shows the same holds for $\inf _{n \in \mathbf{N}_{0}}\left(\frac{m_{n} \cdot n !}{t^{n}}\right)$. Therefore

$$
\left[\sup _{n \in \mathbf{N}_{0}}\left(\frac{t^{n}}{m_{n} \cdot n !}\right)\right]^{-1}=\inf _{n \in \mathbf{N}_{0}}\left(\frac{m_{n} \cdot n !}{t^{n}}\right) .
$$

For a full description of the properties of $t \mapsto \sup _{n \in \mathbf{N}_{0}}\left(\frac{t^{n}}{m_{n} \cdot n !}\right)$, which is called associated function, we recommend the section with similar title in [K1].

Remark 4.7. For the Gevrey case, Theorems 4.2 and 4.5 may be rewritten in the following way: if $u$ is a $s$-Gevrey ultradistribution, then

$$
\forall \varepsilon, \exists C_{\varepsilon}>0 ;|\widehat{u}(\xi)| \leq C_{\varepsilon} \cdot e^{\varepsilon \cdot|\xi|^{1 / s}}, \quad \forall \xi \in \mathbf{Z}^{N} .
$$

In addition, if $\varphi$ is a $s$-Gevrey function, then

$$
\exists C, \delta>0 ;|\widehat{\varphi}(\xi)| \leq C \cdot e^{-\delta \cdot|\xi|^{1 / s}}, \quad \forall \xi \in \mathbf{Z}^{N} .
$$

In fact, it is not difficult to show that chacterizations using $\sup _{n \in \mathbf{N}_{0}}\left(\frac{t^{n}}{n !^{s}}\right)$ and $e^{t^{1 / s}}$ are equivalent.

After these two brief remarks, we turn back to the previous goal.

Theorem 4.8. Let $\left\{a_{\xi}\right\}_{\xi \in \mathbf{Z}^{N}}$ be a sequence of complex numbers satisfying the following condition: for every $\varepsilon>0$ there exists $C_{\varepsilon}>0$ such that

$$
\left|a_{\xi}\right| \leq C_{\varepsilon} \cdot \sup _{n \in \mathbf{N}_{0}}\left(\frac{\varepsilon^{n} \cdot(1+|\xi|)^{n}}{m_{n} \cdot n !}\right), \quad \forall \xi \in \mathbf{Z}^{N} .
$$

Then $u=\sum_{\xi \in \mathbf{Z}^{N}} a_{\xi} \cdot e^{i x \xi}$ belongs to $\mathscr{D}_{\mathscr{M}}^{\prime}\left(\mathbf{T}^{N}\right)$ and $\hat{u}(\xi)=a_{\xi}$.

Proof. Put $s_{j}(x)=\sum_{|\xi| \leq j} a_{\xi} \cdot e^{i x \xi}$, for every $j \in \mathbf{N}$. For any $\varphi \in \mathcal{E}_{\mathscr{M}}\left(\mathbf{T}^{N}\right)$, we claim that

$$
\left\langle s_{j}, \varphi\right\rangle:=\int_{\mathbf{T}^{N}} \sum_{|\xi| \leq j} a_{\xi} \cdot e^{i x \xi} \cdot \varphi(x) d x=\sum_{|\xi| \leq j} a_{\xi} \cdot \int_{\mathbf{T}^{N}} e^{i x \xi} \cdot \varphi(x) d x
$$

is a Cauchy sequence in $\mathbf{C}$. In fact, by taking $m, k \in \mathbf{N}$ with $m>k$,

$$
\left\langle s_{m}-s_{k}, \varphi\right\rangle=(2 \pi)^{N} \sum_{k+1 \leq|\xi| \leq m} a_{\xi} \cdot \hat{\varphi}(-\xi) .
$$


By Theorem 4.2, there exist $C_{1}, \delta>0$ such that

$$
|\hat{\varphi}(\xi)| \leq C_{1} \cdot \inf _{n \in \mathbf{N}_{0}}\left(\frac{m_{n} \cdot n !}{\delta^{n} \cdot(1+|\xi|)^{n}}\right), \quad \forall \xi \in \mathbf{Z}^{N} .
$$

Thus, for some $\varepsilon>0$ which will be chosen later, we have

$$
\left|\left\langle s_{m}-s_{k}, \varphi\right\rangle\right| \leq(2 \pi)^{N} C_{1} C_{\varepsilon} \sum_{k+1 \leq|\xi| \leq m} \sup _{n \in \mathbf{N}_{0}}\left(\frac{\varepsilon^{n} \cdot(1+|\xi|)^{n}}{m_{n} \cdot n !}\right) \inf _{n \in \mathbf{N}_{0}}\left(\frac{m_{n} \cdot n !}{\delta^{n}(1+|\xi|)^{n}}\right) .
$$

On the other hand, it follows from (2.1), (2.2) and Remark 4.6 that

$$
\begin{aligned}
\sup _{n \in \mathbf{N}_{0}}\left(\frac{\varepsilon^{n} \cdot(1+|\xi|)^{n}}{m_{n} \cdot n !}\right) & \inf _{n \in \mathbf{N}_{0}}\left(\frac{m_{n} \cdot n !}{\delta^{n}(1+|\xi|)^{n}}\right)=\left(\frac{\varepsilon^{n_{0}} \cdot(1+|\xi|)^{n_{0}}}{m_{n_{0}} \cdot n_{0} !}\right) \inf _{n \in \mathbf{N}_{0}}\left(\frac{m_{n} \cdot n !}{\delta^{n}(1+|\xi|)^{n}}\right) \\
& \leq\left(\frac{\varepsilon^{n_{0}} \cdot(1+|\xi|)^{n_{0}}}{m_{n_{0}} \cdot n_{0} !}\right)\left(\frac{m_{\left(n_{0}+2 N\right)} \cdot\left(n_{0}+2 N\right) !}{\delta^{n_{0}+2 N} \cdot(1+|\xi|)^{n_{0}+2 N}}\right) \\
& \leq \frac{1}{\delta^{2 N}} \cdot\left(\frac{\varepsilon \cdot B_{\{2 N\}} \cdot C_{\{2 N\}}}{\delta}\right)^{n_{0}} \cdot \frac{1}{(1+|\xi|)^{2 N}},
\end{aligned}
$$

for $n_{0}$ depending on $\xi$ and $\varepsilon$. However, if we choose $\varepsilon=\frac{\delta}{C_{\{2 N\}} \cdot B_{\{2 N\}}}$, the dependence on $n_{0}$ disappears. Hence, if $C_{2}=\frac{(2 \pi)^{N} \cdot C_{\varepsilon} \cdot C_{1}}{\delta^{2 N}}$, we obtain

$$
\left|\left\langle s_{m}-s_{k}, \varphi\right\rangle\right| \leq C_{2} \sum_{k+1 \leq|\xi| \leq m} \frac{1}{(1+|\xi|)^{2 N}}
$$

Since the series on the right-hand side converges, $\lim _{k \rightarrow \infty}\left|\left\langle s_{m}-s_{k}, \varphi\right\rangle\right|=0$, which proves that $\left\langle s_{j}, \varphi\right\rangle$ is a Cauchy sequence for every $\varphi \in \mathcal{E}_{\mathscr{M}}\left(\mathbf{T}^{N}\right)$. By Lemma 3.6, $u:=\sum_{\xi \in \mathbf{Z}^{N}} a_{\xi} \cdot e^{i x \xi}$ is an element of $\mathscr{D}_{\mathscr{M}}^{\prime}\left(\mathbf{T}^{N}\right)$ and it is immediate that $\hat{u}(\xi)=a_{\xi}$.

Theorem 4.9. Let $u \in \mathscr{D}_{\mathscr{M}}^{\prime}\left(\mathbf{T}^{N}\right)$. Then $u=\sum_{\xi \in \mathbf{Z}^{N}} \hat{u}(\xi) \cdot e^{i x \xi}$, with convergence in $\mathscr{D}_{\mathscr{M}}^{\prime}\left(\mathbf{T}^{N}\right)$.

Proof. By Theorems 4.5 and 4.8, $\tilde{u}:=\sum_{\xi \in \mathbf{Z}^{N}} \hat{u}(\xi) \cdot e^{i x \xi}$ satisfies conditions stated. Thus it is sufficient to show that $u=\tilde{u}$. Given $\varphi \in \mathcal{E}_{\mathscr{M}}\left(\mathbf{T}^{N}\right)$, it follows from Theorem 4.2 that $\varphi(x)=\sum_{\xi \in \mathbf{Z}^{N}} \hat{\varphi}(\xi) \cdot e^{i x \xi}$. If we define once again $S_{k} \varphi(x)=$ $\sum_{|\xi| \leq k} \hat{\varphi}(\xi) \cdot e^{i x \xi}$, by using that $S_{k} \varphi \rightarrow \varphi$ in $\mathcal{E}_{\mathscr{M}}\left(\mathbf{T}^{N}\right)$ it is not difficult to check that $\langle u, \varphi\rangle=\langle\tilde{u}, \varphi\rangle$. Therefore $u=\tilde{u}$, which ends the proof.

By associating Theorems 4.3 and 4.9 , we are able to characterize precisely when $u \in \mathscr{D}_{\mathscr{M}}^{\prime}\left(\mathbf{T}^{N}\right)$ is actually an element of $\mathcal{E}_{\mathscr{M}}\left(\mathbf{T}^{N}\right)$ through its Fourier coefficients.

Corollary 4.10. Let $u \in \mathscr{D}_{\mathscr{M}}^{\prime}\left(\mathbf{T}^{N}\right)$; if one can find $C, \delta>0$ such that

$$
|\hat{u}(\xi)| \leq C \cdot \inf _{n \in \mathbf{N}_{0}}\left(\frac{m_{n} \cdot n !}{\delta^{n} \cdot(1+|\xi|)^{n}}\right), \quad \forall \xi \in \mathbf{Z}^{N}
$$

then $u \in \mathcal{E}_{\mathscr{M}}\left(\mathbf{T}^{N}\right)$.

Remark 4.11. The property of moderate growth (Definition 2.1) was not directly used for proofs in this subsection. Only the weaker condition (2.1), proved in Proposition 2.6, was necessary. This fact suggests that those results remain valid for even more general classes of ultradifferentiable functions. 
4.2. Partial Fourier series. Given $R, S \in \mathbf{N}$ such that $R+S=N$, we set $\mathbf{T}^{N}=\mathbf{T}_{t}^{R} \times \mathbf{T}_{x}^{S}$. For any $\varphi \in \mathcal{E}_{\mathscr{M}}\left(\mathbf{T}^{N}\right)$ and $t \in \mathbf{T}^{R}$ fixed, let

$$
\varphi_{t}: \mathbf{T}^{S} \rightarrow \mathbf{C} ; \varphi_{t}(x)=\varphi(t, x) .
$$

Then $\varphi_{t} \in \mathcal{E}_{\mathscr{M}}\left(\mathbf{T}^{S}\right)$ and it follows from Theorem 4.2 that

$$
\varphi(t, x)=\varphi_{t}(x)=\sum_{\eta \in \mathbf{Z}^{S}} \widehat{\varphi}(t, \eta) \cdot e^{i x \eta}
$$

with convergence in $\mathcal{E}_{\mathscr{M}}\left(\mathbf{T}^{S}\right)$ and $\widehat{\varphi}(t, \eta)=\frac{1}{(2 \pi)^{S}} \int_{\mathbf{T}^{S}} \varphi(t, x) \cdot e^{-i x \eta} d x$.

Theorem 4.12. Given $\varphi \in \mathcal{E}_{\mathscr{M}}\left(\mathbf{T}^{N}\right)$, there exists $C, h, \delta>0$ such that

$\left|\partial_{t}^{\alpha} \widehat{\varphi}(t, \eta)\right| \leq C \cdot h^{|\alpha|} \cdot m_{|\alpha|} \cdot|\alpha| ! \cdot \inf _{p \in \mathbf{N}_{0}}\left(\frac{m_{p} \cdot p !}{\delta^{p} \cdot(1+|\eta|)^{p}}\right), \quad \forall t \in \mathbf{T}^{R}, \quad \forall \alpha \in \mathbf{N}_{0}^{R}, \forall \eta \in \mathbf{Z}^{S}$.

Moreover, we have the identity (4.2) with convergence in $\mathcal{E}_{\mathscr{M}}\left(\mathbf{T}^{N}\right)$.

Proof. Since $\partial_{t}^{\alpha} \widehat{\varphi}(t, \eta)=\widehat{\partial_{t}^{\alpha} \varphi}(t, \eta)$, we have $\partial_{t}^{\alpha} \widehat{D_{x}^{\beta}} \varphi(t, \eta)=\eta^{\beta} \cdot \partial_{t}^{\alpha} \widehat{\varphi}(t, \eta)$ and

$$
\begin{aligned}
\left|\eta^{\beta} \cdot \partial_{t}^{\alpha} \widehat{\varphi}(t, \eta)\right| & \leq \sup _{(t, x) \in \mathbf{T}^{N}}\left|\partial_{t}^{\alpha} D_{x}^{\beta} \varphi(t, x)\right| \\
& \leq\|\varphi\|_{\mathscr{M}, h_{1}} \cdot h_{1}^{|\alpha|+|\beta|} \cdot m_{|\alpha|+|\beta|} \cdot(|\alpha|+|\beta|) ! \\
& \leq\|\varphi\|_{\mathscr{M}, h_{1}} \cdot\left[\left(2 h_{1} H\right)^{|\alpha|} \cdot m_{|\alpha|} \cdot|\alpha| !\right] \cdot\left[\left(2 h_{1} H\right)^{|\beta|} \cdot m_{|\beta|} \cdot|\beta| !\right],
\end{aligned}
$$

for some $h_{1}>0$. Given $\eta \neq 0$ and $p \in \mathbf{N}_{0}$, take $\beta \in \mathbf{N}_{0}^{S}$ such that $|\beta|=p$ and $|\eta|^{p} \leq N^{p / 2}\left|\eta^{\beta}\right|$. Then

$$
(1+|\eta|)^{p} \cdot\left|\partial_{t}^{\alpha} \widehat{\varphi}(t, \eta)\right| \leq\|\varphi\|_{\mathscr{M}, h_{1}} \cdot\left[\left(2 h_{1} H\right)^{|\alpha|} \cdot m_{|\alpha|} \cdot|\alpha| !\right] \cdot\left[\left(4 h_{1} H \sqrt{N}\right)^{p} \cdot m_{p} \cdot p !\right] .
$$

Therefore, by taking $h=2 h_{1} H, \delta=\frac{1}{4 h_{1} H \sqrt{N}}$ and $C=\|\varphi\|_{\mathscr{M}, h_{1}} \cdot\left[\inf _{p \in \mathbf{N}_{0}}\left(\frac{m_{p} \cdot p !}{\delta^{p}}\right)\right]^{-1}$, we also include case $\eta=0$ and prove (4.3).

Proceeding to the second statement, put $S_{j} \varphi(t, x)=\sum_{|\eta| \leq j} \widehat{\varphi}(t, \eta) \cdot e^{i x \eta}$. For any $\alpha_{1} \in \mathbf{N}_{0}^{R}$ and $\alpha_{2} \in \mathbf{N}_{0}^{S}$, it follows from (2.1), (2.2) and (4.3) that

$$
\begin{aligned}
& \left|\partial_{t}^{\alpha_{1}} \partial_{x}^{\alpha_{2}}\left(\varphi-S_{j} \varphi\right)(t, x)\right| \\
& \leq \sum_{|\eta|>j} C \cdot h^{\left|\alpha_{1}\right|} \cdot m_{\left|\alpha_{1}\right|} \cdot\left|\alpha_{1}\right| ! \cdot \inf _{p \in \mathbf{N}_{0}}\left(\frac{m_{p} \cdot p !}{\delta^{p} \cdot(1+|\eta|)^{p}}\right) \cdot(1+|\eta|)^{\left|\alpha_{2}\right|} \\
& \leq\left(\frac{C}{\delta^{2 S}} \sum_{|\eta|>j} \frac{1}{(1+|\eta|)^{2 S}}\right) h^{\left|\alpha_{1}\right|}\left(\frac{B_{\{2 S\}} C_{\{2 S\}}}{\delta}\right)^{\left|\alpha_{2}\right|} m_{\left|\alpha_{1}\right|+\left|\alpha_{2}\right|}\left(\left|\alpha_{1}\right|+\left|\alpha_{2}\right|\right) ! \\
& \leq\left(\frac{C}{\delta^{2 S}} \sum_{|\eta|>j} \frac{1}{(1+|\eta|)^{2 S}}\right) h_{2}^{\left|\alpha_{1}\right|+\left|\alpha_{2}\right|} \cdot m_{\left|\alpha_{1}\right|+\left|\alpha_{2}\right|} \cdot\left(\left|\alpha_{1}\right|+\left|\alpha_{2}\right|\right) !,
\end{aligned}
$$

with $h_{2}=\max \left\{h, \frac{B_{\{2 S\}} \cdot C_{\{2 S\}}}{\delta}\right\}$. Therefore $S_{j} \varphi \rightarrow \varphi$ in $\mathcal{E}_{\mathscr{M}, h_{2}}\left(\mathbf{T}^{N}\right)$ and consequently in $\mathcal{E}_{\mathscr{M}}\left(\mathbf{T}^{N}\right)$.

Now we show the converse of Theorem 4.12. 
Theorem 4.13. Consider $\left\{\varphi_{\eta}\right\}_{\eta \in \mathbf{Z}^{S}}$ a sequence in $\mathcal{E}_{\mathscr{M}}\left(\mathbf{T}^{R}\right)$ and suppose the existence of $C, h, \delta>0$ such that $\left|\partial_{t}^{\alpha} \varphi_{\eta}(t)\right| \leq C \cdot h^{|\alpha|} \cdot m_{|\alpha|} \cdot|\alpha| ! \cdot \inf _{p \in \mathbf{N}_{0}}\left(\frac{m_{p} \cdot p !}{\delta^{p} \cdot(1+|\eta|)^{p}}\right), \quad \forall t \in \mathbf{T}^{R}, \quad \forall \alpha \in \mathbf{N}_{0}^{R}, \quad \forall \eta \in \mathbf{Z}^{S}$. Then $\varphi: \mathbf{T}^{N} \rightarrow \mathbf{C} ; \varphi(t, x)=\sum_{\eta \in \mathbf{Z}^{S}} \varphi_{\eta}(t) \cdot e^{i x \eta} \in \mathcal{E}_{\mathscr{M}}\left(\mathbf{T}^{N}\right)$, with convergence in the same space.

Proof. It is immediate that $\varphi$ is well defined. For any $\alpha_{1} \in \mathbf{N}_{0}^{R}$ and $\alpha_{2} \in \mathbf{N}_{0}^{S}$, we have

$$
\begin{aligned}
& \left|\partial_{t}^{\alpha_{1}} \partial_{x}^{\alpha_{2}} \varphi(t, x)\right| \leq \sum_{\eta \in \mathbf{Z}^{S}}\left|\partial_{t}^{\alpha_{1}} \varphi_{\eta}(t)\right| \cdot(1+|\eta|)^{\left|\alpha_{2}\right|} \\
& \leq \frac{C}{\delta^{2 S}} \cdot h^{\left|\alpha_{1}\right|}\left(\frac{B_{\{2 S\}} \cdot C_{\{2 S\}}}{\delta}\right)^{\left|\alpha_{2}\right|} m_{\left|\alpha_{1}\right|+\left|\alpha_{2}\right|} \cdot\left(\left|\alpha_{1}\right|+\left|\alpha_{2}\right|\right) ! \sum_{\eta \in \mathbf{Z}^{S}} \frac{1}{(1+|\eta|)^{2 S}} \\
& \leq C_{1} \cdot h_{1}^{\left|\alpha_{1}\right|+\left|\alpha_{2}\right|} \cdot m_{\left|\alpha_{1}\right|+\left|\alpha_{2}\right|} \cdot\left(\left|\alpha_{1}\right|+\left|\alpha_{2}\right|\right) !
\end{aligned}
$$

by applying (2.1), (2.2), and setting $C_{1}=\frac{C}{\delta^{2 S}} \sum_{\eta \in \mathbf{Z}^{S}} \frac{1}{(1+|\eta|)^{2 S}}, h_{1}=\frac{h \cdot B_{\{2 N\}} \cdot C_{\{2 N\}}}{\delta}$, which shows that $\varphi \in \mathcal{E}_{\mathscr{M}}\left(\mathbf{T}^{N}\right)$. Convergence in $\mathcal{E}_{\mathscr{M}}\left(\mathbf{T}^{N}\right)$ is proved just like in Theorem 4.12.

We now aim to obtain versions of Theorems 4.12 and 4.13 for ultradistributions. Although it is not possible to talk about fixing variables in this context, we can proceed analogously by fixing test functions defined on some variables. Given $u$ in $\mathscr{D}_{\mathscr{M}}^{\prime}\left(\mathbf{T}^{N}\right)$ and $\psi \in \mathcal{E}_{\mathscr{M}}\left(\mathbf{T}^{R}\right)$, set

$$
u_{\psi}: \mathbf{T}^{S} \rightarrow \mathbf{C}, \quad \varphi \mapsto\langle u, \psi \otimes \varphi\rangle,
$$

where $\psi \otimes \varphi(t, x)=\psi(t) \cdot \varphi(x)$.

Lemma 4.14. For every $u \in \mathscr{D}_{\mathscr{M}}\left(\mathbf{T}^{N}\right), \psi \in \mathcal{E}_{\mathscr{M}}\left(\mathbf{T}^{R}\right)$, we have that $u_{\psi} \in$ $\mathscr{D}_{\mathscr{M}}^{\prime}\left(\mathbf{T}^{S}\right)$.

Proof. Let $\left\{\varphi_{n}\right\}_{n \in \mathbf{N}}$ be a sequence in $\mathcal{E}_{\mathscr{M}}\left(\mathbf{T}^{S}\right)$ converging to 0 . Because $u$ is continuous, it is sufficient to show that $\psi \otimes \varphi_{n} \rightarrow 0$ in $\mathcal{E}_{\mathscr{M}}\left(\mathbf{T}^{N}\right)$. By our assumption, one can find $h_{S}>0$ such that

$$
\varphi_{n} \in \mathcal{E}_{\mathscr{M}, h_{S}}\left(\mathbf{T}^{S}\right), \forall n \in \mathbf{N} ; \quad\left\|\varphi_{n}\right\|_{\mathscr{M}, h_{S}} \rightarrow 0 .
$$

On the other hand, there exists $h_{R}>0$ such that $\psi \in \mathcal{E}_{\mathscr{M}, h_{R}}\left(\mathbf{T}^{R}\right)$. Hence

$$
\begin{aligned}
\sup _{(t, x) \in \mathbf{T}^{N}}\left|\partial_{t}^{\alpha} \partial_{x}^{\beta}(\psi(t) \cdot \varphi(x))\right| & \leq \sup _{t \in \mathbf{T}^{R}}\left|\partial_{t}^{\alpha} \psi(t)\right| \cdot \sup _{x \in \mathbf{T}^{S}}\left|\partial_{x}^{\beta} \varphi(x)\right| \\
& \leq\|\psi\|_{\mathscr{M}, h_{R}} \cdot h_{R}^{|\alpha|} \cdot m_{|\alpha|} \cdot|\alpha| ! \cdot\left\|\varphi_{n}\right\|_{\mathscr{M}, h_{S}} \cdot h_{S}^{|\beta|} \cdot m_{|\beta|} \cdot|\beta| !
\end{aligned}
$$

If we set $h=\max \left\{h_{R}, h_{S}\right\}$, it follows from inequality above that

$$
\psi \otimes \varphi_{n} \in \mathcal{E}_{\mathscr{M}, h}\left(\mathbf{T}^{N}\right), \quad \forall n \in \mathbf{N}, \quad \text { and }\left\|\psi \otimes \varphi_{n}\right\|_{\mathscr{M}, h} \leq\|\psi\|_{\mathscr{M}, h_{R}}\left\|\varphi_{n}\right\|_{\mathscr{M}_{, h_{S}}} \rightarrow 0,
$$

as we intended to prove.

As a consequence of Lemma 4.14 and Theorem 4.9, we can write $u_{\psi}=\sum_{\eta \in \mathbf{Z}^{S}} \widehat{u_{\psi}}(\eta)$. $e^{i x \eta}$, with $\widehat{u_{\psi}}(\eta)=\frac{1}{(2 \pi)^{S}}\left\langle u, \psi(t) e^{-i x \eta}\right\rangle$ and convergence in $\mathscr{D}_{\mathscr{M}}^{\prime}\left(\mathbf{T}^{N}\right)$. For each $\eta \in \mathbf{Z}^{S}$, we define the functional

$$
\langle\widehat{u}(t, \eta), \psi\rangle=\widehat{u_{\psi}}(\eta)=\frac{1}{(2 \pi)^{S}}\left\langle u, \psi(t) e^{-i x \eta}\right\rangle .
$$


It follows once again from Lemma (4.14) that $\widehat{u}(t, \eta) \in \mathscr{D}_{\mathscr{M}}^{\prime}\left(\mathbf{T}^{R}\right)$ for every $\eta \in \mathbf{Z}^{S}$.

Theorem 4.15. Let $u \in \mathscr{D} \mathscr{M}\left(\mathbf{T}^{N}\right)$. Then

$$
u=\sum_{\eta \in \mathbf{Z}^{S}} \widehat{u}(t, \eta) \cdot e^{i x \eta}
$$

with convergence in $\mathscr{D}_{\mathscr{M}}^{\prime}\left(\mathbf{T}^{N}\right)$. Moreover, for every $\varepsilon, h>0$ there exists $C_{\varepsilon, h}>0$ such that

$$
|\langle\widehat{u}(t, \eta), \psi\rangle| \leq C_{\varepsilon, h} \cdot\|\psi\|_{\mathscr{M}, h} \cdot \sup _{p \in \mathbf{N}_{0}}\left(\frac{\varepsilon^{p} \cdot(1+|\eta|)^{p}}{m_{p} \cdot p !}\right), \forall \eta \in \mathbf{Z}^{S}, \forall \psi \in \mathcal{E}_{\mathscr{M}, h}\left(\mathbf{T}^{R}\right) .
$$

Proof. Fix $\lambda(t, x) \in \mathcal{E}_{\mathscr{M}}\left(\mathbf{T}^{N}\right)$; it follows from Theorem 4.12 that

$$
\begin{aligned}
\langle u, \lambda\rangle & =\lim _{k \rightarrow+\infty} \sum_{|\eta| \leq k}\left\langle u, \widehat{\lambda}(t, \eta) \cdot e^{i x \eta}\right\rangle \\
& =\lim _{k \rightarrow+\infty} \sum_{|\eta| \leq k}\left\langle\widehat{u}(t, \eta), \int_{\mathbf{T}^{S}} \lambda(t, x) \cdot e^{i x \eta} d x\right\rangle \\
& =\lim _{k \rightarrow+\infty} \sum_{|\eta| \leq k}\left\langle\widehat{u}(t, \eta) \cdot e^{i x \eta}, \lambda\right\rangle .
\end{aligned}
$$

Next we proceed to the proof of (4.4). Given $\varepsilon, h>0$; it follows from Theorem 3.4 the existence of $C_{\varepsilon}$ such that

$$
\begin{aligned}
|\langle\widehat{u}(t, \eta), \psi\rangle| & \leq \frac{C_{\varepsilon}}{(2 \pi)^{S}} \sup _{(\alpha, \beta) \in \mathbf{N}_{0}^{N}}\left(\frac{\sup _{(t, x) \in \mathbf{T}^{N}}\left|\partial_{t}^{\alpha} \partial_{x}^{\beta}\left(\psi(t) \cdot e^{-i x \eta}\right)\right| \cdot \varepsilon^{|\alpha+\beta|}}{m_{|\alpha+\beta|} \cdot(|\alpha+\beta|) !}\right) \\
& \leq \frac{C_{\varepsilon}}{(2 \pi)^{S}}\|\psi\|_{\mathscr{M}, h} \sup _{(\alpha, \beta) \in \mathbf{N}_{0}^{N}}\left(\frac{h^{|\alpha|} \cdot(1+|\eta|)^{|\beta|} \cdot \varepsilon^{|\alpha|} \cdot \varepsilon^{|\beta|}}{m_{|\beta|} \cdot|\beta| !}\right) .
\end{aligned}
$$

When $\varepsilon \leq \frac{1}{h}$, we obtain

$$
\begin{aligned}
|\langle\widehat{u}(t, \eta), \psi\rangle| & \leq \frac{C_{\varepsilon}}{(2 \pi)^{S}}\|\psi\|_{\mathscr{M}, h} \sup _{\beta \in \mathbf{N}_{0}^{S}}\left(\frac{\varepsilon^{|\beta|} \cdot(1+|\eta|)^{|\beta|}}{m_{|\beta|} \cdot|\beta| !}\right) \\
& =\frac{C_{\varepsilon}}{(2 \pi)^{S}}\|\psi\|_{\mathscr{M}, h} \sup _{p \in \mathbf{N}_{0}}\left(\frac{\varepsilon^{p} \cdot(1+|\eta|)^{p}}{m_{p} \cdot p !}\right) .
\end{aligned}
$$

Otherwise,

$$
\begin{aligned}
|\langle\widehat{u}(t, \eta), \psi\rangle| & \leq \frac{C_{1 / h}}{(2 \pi)^{S}}\|\psi\|_{\mathscr{M}, h} \sup _{p \in \mathbf{N}_{0}}\left(\frac{(1+|\eta|)^{p}}{h^{p} \cdot m_{p} \cdot p !}\right) \\
& \leq \frac{C_{1 / h}}{(2 \pi)^{S}}\|\psi\|_{\mathscr{M}, h} \sup _{p \in \mathbf{N}_{0}}\left(\frac{\varepsilon^{p} \cdot(1+|\eta|)^{p}}{m_{p} \cdot p !}\right) .
\end{aligned}
$$

Therefore (4.4) holds by taking $C_{h, \varepsilon}=\max \left\{\frac{C_{\varepsilon}}{(2 \pi)^{S}}, \frac{C_{1 / h}}{(2 \pi)^{S}}\right\}$.

Remark 4.16. The notation set is chosen to be analogous to the one applied for ultradifferentiable functions; if $u \in \mathscr{D}_{\mathscr{M}}^{\prime}\left(\mathbf{T}^{N}\right), \widehat{u}(t, \eta)$ is not necessarily a function.

Before we exhibit the reciprocal of Theorem 4.15, it is necessary to state a result which is a consequence of moderate growth condition and will be of great relevance from now on. 
Lemma 4.17. [K1, Proposition 3.6] Let $H$ be as in condition 3 of Definition 2.1; then

$$
\left[\sup _{n \in \mathbf{N}_{0}}\left(\frac{\rho^{n}}{m_{n} \cdot n !}\right)\right]^{2} \leq \sup _{n \in \mathbf{N}_{0}}\left(\frac{\rho^{n} \cdot(2 H)^{n}}{m_{n} \cdot n !}\right), \quad \forall \rho>0 .
$$

Theorem 4.18. Consider $\left\{u_{\eta}\right\}_{\eta \in \mathbf{Z}^{S}}$ a sequence in $\mathscr{D}_{\mathscr{M}}^{\prime}\left(\mathbf{T}^{R}\right)$ satisfying the following condition: for every $\varepsilon, h>0$, we can find $C_{\varepsilon, h}>0$ such that

$$
\left|\left\langle u_{\eta}, \psi\right\rangle\right| \leq C_{\varepsilon, h}\|\psi\|_{\mathscr{M}, h} \sup _{p \in \mathbf{N}_{0}}\left(\frac{\varepsilon^{p} \cdot(1+|\eta|)^{p}}{m_{p} \cdot p !}\right), \quad \forall \eta \in \mathbf{Z}^{S}, \quad \forall \psi \in \mathcal{E}_{\mathscr{M}, h}\left(\mathbf{T}^{R}\right) .
$$

Then $u:=\sum_{\eta \in \mathbf{Z}^{S}} u_{\eta} \cdot e^{i x \eta}$ is an element of $\mathscr{D}_{\mathscr{M}}^{\prime}\left(\mathbf{T}^{N}\right)$.

Proof. Put $s_{j}=\sum_{|\eta| \leq j} u_{\eta} \cdot e^{i x \eta} ;$ if $\lambda(t, x) \in \mathcal{E}_{\mathscr{M}, h}\left(\mathbf{T}^{N}\right)$, we have

$$
\left\langle s_{j}, \lambda\right\rangle=\sum_{|\eta| \leq j}\left\langle u_{\eta} \cdot e^{i x \eta}, \lambda\right\rangle=\sum_{|\eta| \leq j}\left\langle u_{\eta}, \int_{\mathbf{T}^{S}} \lambda(t, x) \cdot e^{i x \eta} d x\right\rangle .
$$

Hence, if $k \in \mathbf{N}$ it follows from hypothesis and Theorem 4.12 that

$$
\begin{aligned}
\left|\left\langle s_{k+j}-s_{j}, \lambda\right\rangle\right| & \leq(2 \pi)^{S} \sum_{j<|\eta| \leq j+k}\left|\left\langle u_{\eta}, \widehat{\lambda}(t,-\eta)\right\rangle\right| \\
& \leq(2 \pi)^{S} \sum_{j<|\eta| \leq j+k} C_{\kappa, 2 h H} \|\left.\widehat{\lambda}(\cdot,-\eta)\right|_{\mathscr{M}, 2 h H} \sup _{p \in \mathbf{N}_{0}}\left(\frac{\kappa^{p} \cdot(1+|\eta|)^{p}}{m_{p} \cdot p !}\right) \\
& \leq \sum_{j<|\eta| \leq j+k} C_{\kappa, 2 h H}^{\prime} \inf _{p \in \mathbf{N}_{0}}\left(\frac{m_{p} \cdot p !}{\delta^{p} \cdot(1+|\eta|)^{p}}\right) \sup _{p \in \mathbf{N}_{0}}\left(\frac{\kappa^{p} \cdot(1+|\eta|)^{p}}{m_{p} \cdot p !}\right),
\end{aligned}
$$

with $\kappa>0$ which has yet to be defined.

By taking $\kappa=\frac{1}{2 H \delta}$, it follows from Lemma 4.17 that

$$
\begin{aligned}
\left|\left\langle s_{k+j}-s_{j}, \lambda\right\rangle\right| & \leq \sum_{j<|\eta| \leq j+k} C_{\kappa, 2 h H}^{\prime} \inf _{p \in \mathbf{N}_{0}}\left(\frac{m_{p} \cdot p !}{\delta^{p} \cdot(1+|\eta|)^{p}}\right)^{1 / 2} \\
& \leq \sum_{j<|\eta| \leq j+k} C_{\kappa, 2 h H}^{\prime}\left(\frac{\left(m_{4 N} \cdot 4 N !\right)^{1 / 2}}{\delta^{2 N} \cdot(1+|\eta|)^{2 N}}\right) \\
& \leq C_{\kappa, 2 h H}^{\prime \prime} \cdot \sum_{j<|\eta| \leq j+k} \frac{1}{(1+|\eta|)^{2 N}} .
\end{aligned}
$$

Hence $\lim _{j \rightarrow \infty}\left|\left\langle s_{k+j}-s_{j}, \lambda\right\rangle\right|=0$, which shows that $\left\langle s_{j}, \varphi\right\rangle$ is a Cauchy sequence for every $\varphi \in \mathcal{E}_{\mathscr{M}}\left(\mathbf{T}^{N}\right)$. By Lemma 3.6, we conclude that $\lim _{j} s_{j}=u \in \mathscr{D} \mathscr{M}\left(\mathbf{T}^{N}\right)$.

\section{Applications}

5.1. Systems of constant coefficient operators. Generalizing real analytic $[\mathrm{Gr}]$ and Gevrey $[\mathrm{HP}]$ cases, we show in this subsection that global hypoellipticity for systems of constant coefficient operators is described by the assymptotic behavior of 
their symbols. Let $P_{1}(D), P_{2}(D), \cdots, P_{k}(D)$ be linear partial differential operators with constant coefficients acting on $\mathbf{T}^{N}$ and consider the system $\mathcal{P}$ given by

$$
P_{j} u=f_{j}, \quad u \in \mathscr{D}_{\mathscr{M}}^{\prime}\left(\mathbf{T}^{N}\right), \quad f_{j} \in \mathscr{D}_{\mathscr{M}}^{\prime}\left(\mathbf{T}^{N}\right), \quad j=1,2, \ldots, k .
$$

Definition 5.1. We say that $\mathcal{P}$ is globally $\mathscr{M}$-hypoelliptic if $u \in \mathscr{D}_{\mathscr{M}}^{\prime}\left(\mathbf{T}^{N}\right), f_{j} \in \mathcal{E}_{\mathscr{M}}\left(\mathbf{T}^{N}\right) \quad$ and $\quad P_{j}(D) u=f_{j}, \quad j=1,2, \ldots, k \Rightarrow u \in \mathcal{E}_{\mathscr{M}}\left(\mathbf{T}^{N}\right)$.

Theorem 5.2. A system in the form (5.1) is globally $\mathscr{M}$-hypoelliptic if and only if for every $\varepsilon>0$, there exists $R_{\varepsilon}>0$ such that

$$
\max _{1 \leq j \leq k}\left|P_{j}(\xi)\right| \geq \inf _{n \in \mathbf{N}_{0}}\left(\frac{m_{n} \cdot n !}{\varepsilon^{n} \cdot(1+|\xi|)^{n}}\right), \quad \forall \xi \in \mathbf{Z}^{N} ;|\xi| \geq R_{\varepsilon}
$$

where $P_{j}(\xi)$ denotes the symbol of $P_{j}(D)$.

Proof. We start by proving the sufficiency: let $u \in \mathscr{D}_{\mathscr{M}}^{\prime}\left(\mathbf{T}^{N}\right)$ such that

$$
P_{j}(D) u=f_{j} \in \mathcal{E}_{\mathscr{M}}\left(\mathbf{T}^{N}\right), \quad \text { for } j=1,2, \ldots, k .
$$

It follows from Theorem 4.2 the existence of $C, \delta>0$ satisfying

$$
\left|\widehat{f}_{j}(\xi)\right| \leq C \cdot \inf _{n \in \mathbf{N}_{0}}\left(\frac{m_{n} \cdot n !}{\delta^{n} \cdot(1+|\xi|)^{n}}\right), \quad \forall \xi \in \mathbf{Z}^{N}, \quad j=1,2, \ldots, k .
$$

By Theorem 4.9, $u=\sum_{\xi \in \mathbf{Z}^{N}} \widehat{u}(\xi) e^{i x \xi}$. Put $\varepsilon=\frac{\delta}{2 H}$; it follows from Lemma 4.17 and (5.2) that

$$
\begin{aligned}
|\widehat{u}(\xi)| & \leq C \inf _{n \in \mathbf{N}_{0}}\left(\frac{m_{n} \cdot n !}{\delta^{n} \cdot(1+|\xi|)^{n}}\right) \sup _{n \in \mathbf{N}_{0}}\left(\frac{(\delta / 2 H)^{n}(1+|\xi|)^{n}}{m_{n} \cdot n !}\right) \\
& \leq C \inf _{n \in \mathbf{N}_{0}}\left(\frac{m_{n} \cdot n !}{\varepsilon^{n}(1+|\xi|)^{n}}\right), \quad|\xi| \geq R_{\delta / 2 H} .
\end{aligned}
$$

Thus by possibly increasing $\mathrm{C}$ we conclude that

$$
|\widehat{u}(\xi)| \leq C \cdot \inf _{n \in \mathbf{N}_{0}}\left(\frac{m_{n} \cdot n !}{\varepsilon^{n} \cdot(1+|\xi|)^{n}}\right), \forall \xi \in \mathbf{Z}^{N},
$$

which allows us to infer (Corollary 4.10) that $u \in \mathcal{E}_{\mathscr{M}}\left(\mathbf{T}^{N}\right)$.

On the other hand, if (5.2) does not hold there exist $\kappa>0$ and $\left\{\xi_{\ell}\right\}_{\ell \in \mathbf{N}}$ in $\mathbf{Z}^{N}$ such that

$$
\max _{1 \leq j \leq k}\left|P_{j}\left(\xi_{\ell}\right)\right|<\inf _{n \in \mathbf{N}_{0}}\left(\frac{m_{n} \cdot n !}{\kappa^{n} \cdot\left(1+\left|\xi_{\ell}\right|\right)^{n}}\right) \quad \text { and }\left|\xi_{\ell}\right| \geq \ell, \quad \forall \ell \in \mathbf{N} .
$$

Put $u:=\sum_{\ell \in \mathbf{N}} e^{i x \cdot \xi_{\ell}}$, which is an ultradistribution (Theorem 4.8), but not a smooth function. Then $f_{j}:=P_{j}(D) u=\sum_{\ell \in \mathbf{N}} P_{j}\left(\xi_{\ell}\right) \cdot e^{i x \cdot \xi_{\ell}}$ and

$$
\left|P_{j}\left(\xi_{\ell}\right)\right| \leq \max _{1 \leq q \leq k}\left|P_{q}\left(\xi_{\ell}\right)\right|<\inf _{n \in \mathbf{N}_{0}}\left(\frac{m_{n} \cdot n !}{\kappa^{n} \cdot\left(1+\left|\xi_{\ell}\right|\right)^{n}}\right), \forall \ell \in \mathbf{N}, \quad j=1,2, \ldots, k .
$$

It follows from Corollary 4.10 that $f_{j} \in \mathcal{E}_{\mathscr{M}}\left(\mathbf{T}^{N}\right)$ for $j=1,2, \ldots, k$, as we intended to prove.

Corollary 5.3. Suppose that $\mathcal{P}$ described in (5.1) is globally $C^{\infty}$-hypoelliptic. Then it is also globally $\mathscr{M}$-hypoelliptic. 
Proof. By Greenfield-Wallach's condition ([GW]), global smooth hypoellipticity of $\mathcal{P}$ implies the existence of $L, \ell, R>0$ such that

$$
\max _{1 \leq j \leq k}\left|P_{j}(\xi)\right| \geq \frac{L}{(1+|\xi|)^{\ell}}, \quad|\xi| \geq R
$$

Note that we may consider $\ell \in \mathbf{N}$. Given $\varepsilon>0$, we take $R_{\varepsilon}=\max \left\{R, \frac{m_{\ell+1} \cdot(\ell+1) !}{L \cdot \varepsilon^{\ell+1}}\right\}$ and obtain

$$
\begin{aligned}
|\xi| \geq R_{\varepsilon} \Rightarrow \max _{1 \leq j \leq k}\left|P_{j}(\xi)\right| & \geq \frac{L}{(1+|\xi|)^{\ell}}=\frac{L \cdot(1+|\xi|)}{(1+|\xi|)^{\ell+1}} \geq \frac{m_{\ell+1} \cdot(\ell+1) !}{\varepsilon^{\ell+1} \cdot(1+|\xi|)^{\ell+1}} \\
& \geq \inf _{n \in \mathbf{N}_{0}}\left(\frac{m_{n} \cdot n !}{\varepsilon^{n} \cdot(1+|\xi|)^{n}}\right),
\end{aligned}
$$

which ends our proof.

Corollary 5.4. Let $\mathscr{M}$ and $\mathscr{L}$ be weight sequences such that $\mathcal{E}_{\mathscr{M}}\left(\mathbf{T}^{N}\right) \subset \mathcal{E}_{\mathscr{L}}\left(\mathbf{T}^{N}\right)$. If the system (5.1) is globally $\mathscr{L}$-hypoelliptic, it is also globally $\mathscr{M}$-hypoelliptic.

Proof. By (2.3) and (2.4), there exists $C \geq 1$ such that

$$
m_{k} \leq C^{k} \cdot \ell_{k}, \forall k \in \mathbf{N}_{0}
$$

By hypothesis, for any $\varepsilon>0$ we are able to find $R_{\varepsilon}$ satisfying

$\max _{1 \leq j \leq k}\left|P_{j}(\xi)\right| \geq \inf _{n \in \mathbf{N}_{0}}\left(\frac{C^{n} \cdot \ell_{n} \cdot n !}{\varepsilon^{n} \cdot(1+|\xi|)^{n}}\right) \geq \inf _{n \in \mathbf{N}_{0}}\left(\frac{m_{n} \cdot n !}{\varepsilon^{n} \cdot(1+|\xi|)^{n}}\right), \quad \forall \xi \in \mathbf{Z}^{N} ;|\xi| \geq R_{\varepsilon}$ which proves our assertion.

5.2. Greenfield-Wallach vector fields. Similarly to what was done in [Gr], [GW] and [GPY], we may apply Theorem 5.2 to study global $\mathscr{M}$-hypoellipticity for the following vector field acting on $\mathbf{T}^{2}$ :

$$
P_{\alpha}=\frac{\partial}{\partial t}-\alpha \frac{\partial}{\partial x}, \quad \alpha \in \mathbf{C} .
$$

If $\alpha$ is not a real number, $P_{\alpha}$ is elliptic and it follows from Corollary 5.3 that it is globally $\mathscr{M}$-hypoelliptic for any weight sequence $\mathscr{M}$. Hence the interesting cases occur when $\alpha \in \mathbf{R}$.

Definition 5.5. We say $\alpha \in \mathbf{R} \backslash \mathbf{Q}$ is $\mathscr{M}$-exponential Liouville if there exists $\varepsilon>0$ such that inequality

$$
|\xi-\alpha \eta|<\inf _{n \in \mathbf{N}_{0}}\left(\frac{m_{n} \cdot n !}{\varepsilon^{n} \cdot(1+|\eta|)^{n}}\right), \quad(\xi, \eta) \in \mathbf{Z} \times \mathbf{Z},
$$

has infinite solutions.

Proposition 5.6. Let $\alpha$ be a real number; then $P_{\alpha}$ is globally $\mathscr{M}$-hypoelliptic if and only if $\alpha$ is irrational and not $\mathscr{M}$-exponential Liouville.

Proof. Since $P_{\alpha}(\xi, \eta)=-i(\xi-\alpha \eta)$ for $(\xi, \eta) \in \mathbf{Z} \times \mathbf{Z}$, when $\alpha \in \mathbf{Q}$ it is possible to obtain a sequence $\left\{\xi_{m}, \eta_{m}\right\}_{m \in \mathbf{N}}$ such that $P_{\alpha}\left(\xi_{m}, \eta_{m}\right)=0$ for every $m$, which implies that $P_{\alpha}$ is not globally $\mathscr{M}$-hypoelliptic, by Theorem 5.2.

We proceed to the case where $\alpha$ is irrational; if $\alpha$ is not $\mathscr{M}$-exponential Liouville, for every $\varepsilon>0$, one can find $R_{\varepsilon}>0$ such that

$$
|\xi-\alpha \eta| \geq \inf _{n \in \mathbf{N}_{0}}\left(\frac{m_{n} \cdot n !}{\varepsilon^{n} \cdot(1+|\xi|+|\eta|)^{n}}\right), \quad|\xi|+|\eta| \geq R_{\varepsilon},
$$


proving that $P_{\alpha}$ is globally $\mathscr{M}$-hypoelliptic (Theorem 5.2).

On the other hand, suppose $P_{\alpha}$ globally $\mathscr{M}$-hypoelliptic; it follows from Theorem 5.2 that the right-hand side in (5.4) holds. For any $\delta>0$, put $\varepsilon=\frac{\delta}{(|\alpha|+3)}$; when $|\xi-\alpha \eta|>1$, we have

$$
|\xi-\alpha \eta|>1 \geq \inf _{n \in \mathbf{N}_{0}}\left(\frac{m_{n} \cdot n !}{\varepsilon^{n} \cdot(1+|\eta|)^{n}}\right) .
$$

Otherwise $|\xi|-|\alpha| \cdot|\eta| \leq 1 \Rightarrow|\xi| \leq|\alpha| \cdot|\eta|+1$. Hence when $|\xi|+|\eta| \geq R_{\varepsilon}$, it follows that

$$
|\xi-\alpha \eta| \geq \inf _{n \in \mathbf{N}_{0}}\left(\frac{m_{n} \cdot n !}{(\varepsilon \cdot(|\alpha|+3))^{n} \cdot(|\eta|)^{n}}\right) \geq \inf _{n \in \mathbf{N}_{0}}\left(\frac{m_{n} \cdot n !}{\delta^{n} \cdot(1+|\eta|)^{n}}\right)
$$

since we may consider $\eta \neq 0$, showing that $\alpha$ is not $\mathscr{M}$-exponential Liouville.

It was proved in Corollary 5.4 that if $\mathscr{M}, \mathscr{L}$ are weight sequences, then $\mathcal{E}_{\mathscr{M}}\left(\mathbf{T}^{N}\right) \subset \mathcal{E}_{\mathscr{L}}\left(\mathbf{T}^{N}\right)$ and $P_{\alpha}$ is globally $\mathscr{L}$-hypoelliptic, then $P_{\alpha}$ is also globally $\mathscr{M}$ hypoelliptic. On the other hand, it is demonstrated in [GPY] that for any $1 \leq r<s$ there exists $\alpha \in \mathbf{R} \backslash \mathbf{Q}$ such that $P_{\alpha}$ is globally $\mathcal{G}^{r}$-hypoelliptic, but it is not globally $\mathcal{G}^{s}$-hypoelliptic. Next we extend those results. denote

Definition 5.7. Let $\mathscr{L}=\left\{\ell_{n}\right\}_{n \in \mathbf{N}_{0}}, \mathscr{M}=\left\{m_{n}\right\}_{n \in \mathbf{N}_{0}}$ be weight sequences. We

$$
\mathscr{M} \prec \mathscr{L} \Leftrightarrow \lim _{k \rightarrow+\infty}\left(\frac{m_{k}}{\ell_{k}}\right)^{1 / k}=0 .
$$

By notation set in (2.3), when $\mathscr{M} \prec \mathscr{L}$ it implies that $\mathscr{M} \preceq \mathscr{L}$ and $\mathscr{L} \npreceq \mathscr{M}$. We now prove a technical result related to the associated functions of $\mathscr{L}$ and $\mathscr{M}$.

Lemma 5.8. If $\mathscr{M} \prec \mathscr{L}$,

$$
\lim _{t \rightarrow+\infty}\left[\sup _{p \in \mathbf{N}_{0}}\left(\frac{t^{p}}{\ell_{p} \cdot p !}\right) \cdot \inf _{p \in \mathbf{N}_{0}}\left(\frac{m_{p} \cdot p !}{\delta^{p} \cdot t^{p}}\right)\right]=0 \quad \text { for any } \delta>0 .
$$

Proof. Given $\delta>0$, take $H$ as in Definition 2.1 and $q \in \mathbf{N}$ satisfying $\frac{1}{(2 H)^{q}} \leq \delta$. By hypothesis, $\lim _{k \rightarrow+\infty}\left(\frac{\ell_{k}}{m_{k}}\right)^{1 / k}=+\infty$; thus there exists $k_{0} \in \mathbf{N}$ such that

$$
\left(\frac{\ell_{k}}{m_{k}}\right)^{1 / k} \geq(2 H)^{q+1}, \quad \forall k \geq k_{0}
$$

Suppose that $t \geq \ell_{k_{0}} \cdot k_{0}$ !. When $s<k_{0}$ we have

$$
\frac{t^{k_{0}}}{\ell_{k_{0}} \cdot k_{0} !} \div \frac{t^{s}}{\ell_{s} \cdot s !}=t^{k_{0}-s} \cdot \frac{\ell_{s} \cdot s !}{\ell_{k_{0}} \cdot k_{0} !} \geq\left[\ell_{k_{0}} \cdot k_{0} !\right]^{k_{0}-s-1} \cdot \ell_{s} \cdot s ! \geq 1 .
$$

Hence, if $t \geq \ell_{k_{0}} \cdot k_{0}$ ! it follows from (5.5) and (5.6) that

$$
\sup _{p \in \mathbf{N}_{0}}\left(\frac{t^{p}}{\ell_{p} \cdot p !}\right)=\sup _{p \geq k_{0}}\left(\frac{t^{p}}{\ell_{p} \cdot p !}\right) \leq \sup _{p \in \mathbf{N}_{0}}\left(\frac{t^{p}}{\left((2 H)^{q+1}\right)^{p} \cdot m_{p} \cdot p !}\right) .
$$

By Lemma 4.17, we obtain

$$
\begin{aligned}
\sup _{p \in \mathbf{N}_{0}}\left(\frac{t^{p}}{\left((2 H)^{q+1}\right)^{p} \cdot m_{p} \cdot p !}\right) & \leq\left[\sup _{p \in \mathbf{N}_{0}}\left(\frac{t^{p}}{\left((2 H)^{q}\right)^{p} \cdot m_{p} \cdot p !}\right)\right]^{1 / 2} \\
& \leq\left[\sup _{p \in \mathbf{N}_{0}}\left(\frac{\delta^{p} \cdot t^{p}}{m_{p} \cdot p !}\right)\right]^{1 / 2} .
\end{aligned}
$$


By associating (5.7) to (5.8), we deduce that

$$
\begin{aligned}
\sup _{p \in \mathbf{N}_{0}}\left(\frac{t^{p}}{\ell_{p} \cdot p !}\right) \inf _{p \in \mathbf{N}_{0}}\left(\frac{m_{p} \cdot p !}{\delta^{p} \cdot t^{p}}\right) & \leq\left[\sup _{p \in \mathbf{N}_{0}}\left(\frac{\delta^{p} \cdot t^{p}}{m_{p} \cdot p !}\right)\right]^{1 / 2} \inf _{p \in \mathbf{N}_{0}}\left(\frac{m_{p} \cdot p !}{\delta^{p} \cdot t^{p}}\right) \\
& \leq \frac{\sqrt{2 m_{2}}}{\delta t} \text { if } t \geq \ell_{k_{0}} \cdot k_{0} !
\end{aligned}
$$

which proves the assertion.

Theorem 5.9. Let $\mathscr{L}, \mathscr{M}$ be weight sequences such that $\mathscr{M} \prec \mathscr{L}$ and $P_{\alpha}$ a vector field as in (5.3).

1. When $P_{\alpha}$ is globally $\mathscr{L}$-hypoelliptic, it is also globally $\mathscr{M}$-hypoelliptic.

2. There exists $\beta \in \mathbf{R} \backslash \mathbf{Q}$ such that $P_{\beta}$ is globally $\mathscr{M}$-hypoelliptic, but it is not globally $\mathscr{L}$-hypoelliptic.

Proof. The proof of 1 follows immediately from Corollary 5.4. In order to prove 2 , we use $[\mathrm{HW}]$ as a reference for theory of continued fractions. We will exhibit $\beta=\left[a_{0}, a_{1}, \ldots, a_{n}, \ldots\right]$ in the interval $(0,1)$ satisfying the conditions required.

According to Theorem $149 \mathrm{im}[\mathrm{HW}]$, we introduce $\left\{p_{n}\right\}_{n \in \mathbf{N}_{0}},\left\{q_{n}\right\}_{n \in \mathbf{N}_{0}}$ as

$$
\begin{array}{llll}
p_{0}=0, & p_{1}=1, & p_{n}=a_{n} \cdot p_{n-1}+p_{n-2} & (2 \leq n) . \\
q_{0}=1, & q_{1}=0, & q_{n}=a_{n} \cdot q_{n-1}+q_{n-2} & (2 \leq n) .
\end{array}
$$

We also denote (see Section 10.9) $\left\{a_{n}^{\prime}\right\}_{n \in \mathbf{N}_{0}}$ as

$$
a_{n}^{\prime}=\left[a_{n}, a_{n+1}, \ldots\right], \quad \forall n \in \mathbf{N}_{0} .
$$

Next, we list some results in $[\mathrm{HW}]$ :

(i) (Theorems 155 and 156) For $n>3, q_{n+1} \geq q_{n} \geq n$. Thus $\lim _{n \rightarrow \infty} q_{n}=+\infty$.

(ii) (Theorem 168) For every $n \in \mathbf{N}_{0},\left\lfloor a_{n}^{\prime}\right\rfloor=a_{n}$, where $\lfloor$.$\rfloor is the floor function.$

(iii) (Theorem 171) For every $n \geq 1,\left|p_{n}-\beta \cdot q_{n}\right|=\frac{1}{a_{n+1}^{\prime} \cdot q_{n}+q_{n-1}}$. Hence $\left|p_{n}-\beta \cdot q_{n}\right|$ is strictly decreasing and goes to 0 as $n \rightarrow+\infty$.

(iv) (Theorem 182) Let $p \in \mathbf{Z}, q \in \mathbf{N}$ such that $g c d(p, q)=1$ and $q_{k} \leq q<q_{k+1}$. Then

$$
|p-q \cdot \beta| \geq\left|p_{k}-q_{k} \cdot \beta\right|>\left|p_{k+1}-q_{k+1} \cdot \beta\right| .
$$

Put $a_{0}=0$ and assume $a_{j}$ set for $0 \leq j \leq n-1$. It follows from (5.9) that $p_{j}, q_{j}$ are well defined for $j \leq n-1$. Take then

$$
a_{n}= \begin{cases}\left\lfloor\sup _{r \in \mathbf{N}_{0}}\left(\frac{\left(q_{n-1}+1\right)^{r}}{\ell_{r} \cdot r !}\right) / q_{n-1}\right\rfloor, & n \neq 2 . \\ 1, & n=2 .\end{cases}
$$

We claim that if $\beta=\left[a_{0}, a_{1}, \ldots, a_{n}, \ldots\right]$, then $P_{\beta}$ is globally $\mathscr{M}$-hypoelliptic. Let $p \in \mathbf{Z}, q \in \mathbf{N}$ such that $g c d(p, q)=1$. By (i), there exists $k_{0} \in \mathbf{N}$ such that $q_{k_{0}} \leq q<q_{k_{0}+1}$. From (iii) and (iv), we obtain:

$$
|p-q \cdot \beta| \geq\left|p_{k_{0}}-q_{k_{0}} \cdot \beta\right|=\frac{1}{a_{k_{0}+1}^{\prime} \cdot q_{k_{0}}+q_{k_{0}-1}} .
$$


On the other hand, if $q \geq q_{5}$, we infer from (ii) and definition of $\left\{a_{n}\right\}_{n \in \mathbf{N}_{0}}$ that

$$
\begin{aligned}
a_{k_{0}+1}^{\prime} \cdot q_{k_{0}}+q_{k_{0}-1} & =\left\lfloor\sup _{r \in \mathbf{N}_{0}}\left(\frac{\left(q_{k_{0}}+1\right)^{r}}{\ell_{r} \cdot r !}\right) / q_{k_{0}}\right\rfloor \cdot q_{k_{0}}+q_{k_{0}-1} \\
& \leq 3 \sup _{r \in \mathbf{N}_{0}}\left(\frac{(q+1)^{r}}{\ell_{r} \cdot r !}\right) .
\end{aligned}
$$

Hence, it follows from (5.10) that

$$
|p-q \cdot \beta| \geq \frac{1}{3} \inf _{r \in \mathbf{N}_{0}}\left(\frac{\ell_{r} \cdot r !}{(q+1)^{r}}\right) .
$$

We now fix $\delta>0$; by Lemma 5.8 there exists $s \in \mathbf{N}$, which we may consider greater than $q_{5}$, such that

$$
\inf _{r \in \mathbf{N}_{0}}\left(\frac{m_{r} \cdot r !}{\delta^{r} \cdot t^{r}}\right) \leq \frac{1}{3} \cdot \inf _{r \in \mathbf{N}_{0}}\left(\frac{\ell_{r} \cdot r !}{t^{r}}\right), \quad t \geq s .
$$

By associating (5.11) to (5.12), we get

$$
|p-q \cdot \beta| \geq \inf _{r \in \mathbf{N}_{0}}\left(\frac{m_{r} \cdot r !}{\delta^{r} \cdot(1+q)^{r}}\right), \quad \forall q \geq s .
$$

Suppose then that $|p|+|q| \geq 2 s$. If $q \geq s$, we apply (5.13). Otherwise,

$$
|p-q \cdot \beta| \geq|p|-|q| \geq(s+1)-(s-1)=2>\inf _{r \in \mathbf{N}_{0}}\left(\frac{m_{r} \cdot r !}{\delta^{r} \cdot(1+q)^{r}}\right) .
$$

Therefore it follows from Proposition 5.6 that $P_{\beta}$ is globally $\mathscr{M}$-hypoelliptic.

In order to check that $P_{\beta}$ is not globally $\mathscr{L}$-hypoelliptic, we estimate $\left|p_{k_{0}}-q_{k_{0}} \beta\right|$ from below:

$$
\begin{aligned}
a_{k_{0}+1}^{\prime} \cdot q_{k_{0}}+q_{k_{0}-1} & =\left\lfloor\sup _{r \in \mathbf{N}_{0}}\left(\frac{\left(q_{k_{0}}+1\right)^{r}}{\ell_{r} \cdot r !}\right) / q_{k_{0}}\right\rfloor \cdot q_{k_{0}}+q_{k_{0}-1} \\
& >\sup _{r \in \mathbf{N}_{0}}\left(\frac{\left(q_{k_{0}}+1\right)^{r}}{\ell_{r} \cdot r !}\right)-q_{k_{0}} .
\end{aligned}
$$

With a very similar argument to the one applied in Lemma 5.8, one can prove that

$$
\lim _{t \rightarrow+\infty}\left[\sup _{r \in \mathbf{N}_{0}}\left(\frac{t^{r}}{\ell_{r} \cdot r !}\right) \cdot 1 / t\right]=+\infty
$$

Thence it follows from (i) and (5.14) the existence of $d \in \mathbf{N}$ such that

$$
k_{0} \geq d \Rightarrow a_{k_{0}+1}^{\prime} \cdot q_{k_{0}}+q_{k_{0}-1}>\frac{1}{2} \cdot \sup _{r \in \mathbf{N}_{0}}\left(\frac{\left(q_{k_{0}}+1\right)^{r}}{\ell_{r} \cdot r !}\right) .
$$

By (iii) and (5.15), we obtain

$$
\left|p_{n}-q_{n} \cdot \beta\right|<2 \inf _{r \in \mathbf{N}_{0}}\left(\frac{\ell_{r} \cdot r !}{\left(1+q_{n}\right)^{r}}\right), \forall n \geq d .
$$

It is not difficult to see that (5.16) implies $\beta$ is $\mathscr{L}$-exponential Liouville, as we intended to prove.

5.3. Global $\mathscr{M}$-hypoellipticity for a class of systems of real vector fields. Let $N \in \mathbf{N}$ and $\mathcal{L}$ be the system of $N$ vector fields acting on the $(N+1)$ dimensional torus $\mathbf{T}_{t}^{N} \times \mathbf{T}_{x}$ given by

$$
L_{j}=\frac{\partial}{\partial t_{j}}+a^{j}(t) \frac{\partial}{\partial x}, \quad(j=1,2, \ldots, N),
$$


where each $a^{j}$ is a real-valued element of $\mathcal{E}_{\mathscr{M}}\left(\mathbf{T}^{N}\right)$ and

$$
\frac{\partial a^{j}}{\partial t_{k}}=\frac{\partial a^{k}}{\partial t_{j}}, \quad \forall j, k \in\{1,2, \ldots, N\} .
$$

That is, the 1-form $a=\sum_{j=1}^{N} a^{j} d t_{j}$ is closed.

Given $f \in \mathcal{E}_{\mathscr{M}}\left(\mathbf{T}^{N}\right)$, consider

$$
f_{j_{0}}=\int_{0}^{2 \pi} f\left(0, \ldots, 0, t_{j}, 0, \ldots, 0\right) d t_{j} .
$$

We prove in this subsection that $\mathcal{L}$ is globally $\mathscr{M}$-hypoelliptic if and only if the same holds for the system $\widetilde{\mathcal{L}}$ composed by the vector fields

$$
\widetilde{L_{j}}=\frac{\partial}{\partial t_{j}}+a_{j_{0}}^{j} \frac{\partial}{\partial x}, \quad(j=1,2, \ldots, N),
$$

with $a_{j_{0}}^{j}$ as in (5.19). Since $\widetilde{\mathcal{L}}$ has constant coefficients, it follows from Theorem 5.2 that we have covered global $\mathscr{M}$-hypoellipticity for $\mathcal{L}$.

Set $\alpha(t)=\left(\alpha^{1}(t), \ldots, \alpha^{N}(t)\right)$, where

$$
\alpha^{j}: \mathbf{T}^{N} \rightarrow \mathbf{R} ; \quad \alpha^{j}(t)=a^{j}(t)-a_{j_{0}}^{j}, \text { for } j=1,2, \ldots, N .
$$

Note that (5.18) still holds for $\alpha$; furthermore, we have

$$
\alpha_{j_{0}}^{j}=0, \quad \forall j \in\{1,2, \ldots, N\} .
$$

Therefore there exists $\mathcal{E}_{\mathscr{M}}\left(\mathbf{T}^{N} ; \mathbf{R}\right)$ such that

$$
\frac{\partial A}{\partial t_{k}}(t)=\alpha_{k}(t), \quad k=1,2, \ldots, N .
$$

We now move on to describe an important inequality in this subsection. Before that, we need a technical result related to weight sequences.

Lemma 5.10. [BM, Corollary 4.5] Let $k_{1}, \ldots, k_{\ell} \in \mathbf{N}^{p} \backslash\{0\}$ and $\delta_{1}, \ldots, \delta_{\ell}$ in $\mathbf{N}^{n} \backslash\{0\}$, with $\ell, p, n \in \mathbf{N}$. Set $\beta=k_{1}+\ldots+k_{\ell}$ and $\gamma=\left|k_{1}\right| \delta_{1}+\ldots+\left|k_{\ell}\right| \delta_{\ell}$. Then

$$
m_{|\beta|} \cdot m_{\left|\delta_{1}\right|}^{\left|k_{1}\right|} \ldots \cdot m_{\left|\delta_{\ell}\right|}^{\left|k_{\ell}\right|} \leq m_{|\gamma|} .
$$

Proposition 5.11. Let $A$ be as in (5.21); for every $\varepsilon>0$, there exist $C_{\varepsilon}, h_{\varepsilon}>0$ such that

$$
\inf _{p \in \mathbf{N}_{0}}\left(\frac{m_{p} \cdot p !}{\varepsilon^{p} \cdot(1+|\eta|)^{p}}\right) \cdot\left|\partial_{t}^{\alpha} e^{i \eta A(t)}\right| \leq C_{\varepsilon} \cdot h_{\varepsilon}^{|\alpha|} \cdot m_{|\alpha|} \cdot|\alpha| !, \quad \forall t \in \mathbf{T}^{N}, \forall \eta \in \mathbf{Z}, \forall \alpha \in \mathbf{N}_{0}^{N} .
$$

Proof. It is immediate that $A \in \mathcal{E}_{\mathscr{M}}\left(\mathbf{T}^{N}\right)$; hence one can find $C, h>1$ satisfying

$$
\left|\partial_{t}^{\alpha} A(t)\right| \leq C \cdot h^{|\alpha|} \cdot m_{|\alpha|} \cdot|\alpha| !, \quad \forall t \in \mathbf{T}^{N}, \quad \forall \alpha \in \mathbf{N}_{0}^{N} .
$$

In order to estimate the derivatives of $e^{i \eta A(t)}$ we need Faà di Bruno's formula, which will be applied following Proposition 4.3 of $[\mathrm{BM}]$. If $f_{\eta}: \mathbf{R} \rightarrow \mathbf{R} ; f_{\eta}(x)=e^{i \eta x}$, it follows that

$$
\begin{aligned}
& D_{t}^{\alpha}\left(e^{i \eta A(t)}\right) \\
& =\sum \frac{\alpha !}{k_{1} ! \cdot \ldots \cdot k_{\ell} !} \cdot f_{\eta}^{\left(k_{1}+\ldots+k_{\ell}\right)}(A(t)) \cdot\left(\frac{D_{t}^{\delta_{1}} A(t)}{\delta_{1} !}\right)^{k_{1}} \cdot \ldots \cdot\left(\frac{D_{t}^{\delta_{\ell}} A(t)}{\delta_{\ell} !}\right)^{k_{\ell}},
\end{aligned}
$$


with the sum being taken over sets $\left\{\delta_{1}, \ldots, \delta_{\ell}\right\}$ of $\ell$ distinct elements of $\mathbf{N}^{N} \backslash\{0\}$ and $\left(k_{1}, \ldots, k_{\ell}\right)$ in $\mathbf{N}^{\ell}$, with $\ell=1,2,3, \ldots$ and such that

$$
\alpha=\sum_{j=1}^{\ell} k_{j} \delta_{j}
$$

Next we estimate the absolute value of (5.22):

$$
\begin{aligned}
& \left|D_{t}^{\alpha}\left(e^{i \eta A(t)}\right)\right| \\
\leq & \sum \frac{\alpha !}{k_{1} ! \cdot \ldots \cdot k_{\ell} !}|\eta|^{\left(k_{1}+\ldots+k_{\ell}\right)}\left(\frac{C h^{\left|\delta_{1}\right|} m_{\left|\delta_{1}\right|}\left|\delta_{1}\right| !}{\delta_{1} !}\right)^{k_{1}} \cdot \ldots \cdot\left(\frac{C h^{\left|\delta_{\ell}\right|} m_{\left|\delta_{\ell}\right|}\left|\delta_{\ell}\right| !}{\delta_{\ell} !}\right)^{k_{\ell}} \\
\leq & \sum \frac{\alpha !}{k_{1} ! \cdot \ldots \cdot k_{\ell} !}(C|\eta|)^{\left(k_{1}+\ldots+k_{\ell}\right)} \cdot h^{k_{1}\left|\delta_{1}\right|+\ldots+k_{\ell}\left|\delta_{\ell}\right|} \cdot m_{\left|\delta_{1}\right|}^{k_{1}} \times \\
& \ldots \times m_{\left|\delta_{\ell}\right|}^{k_{\ell}} \cdot N^{k_{1}\left|\delta_{1}\right|+\ldots+k_{\ell}\left|\delta_{\ell}\right|} \\
\leq & (N h)^{|\alpha|} \cdot|\alpha| ! \cdot \sum \frac{1}{k_{1} ! \cdot \ldots \cdot k_{\ell} !} \cdot[C(1+|\eta|)]^{\left(k_{1}+\ldots+k_{\ell}\right)} \cdot m_{\left|\delta_{1}\right|}^{k_{1}} \cdot \ldots \cdot m_{\left|\delta_{\ell}\right|}^{k_{\ell}} .
\end{aligned}
$$

Let $\varepsilon>0$ and $(\star):=\inf _{p \in \mathbf{N}_{0}}\left(\frac{m_{p} \cdot p !}{\varepsilon^{p} \cdot(1+|\eta|)^{p}}\right)\left|\partial_{t}^{\alpha} e^{i \eta A(t)}\right|$; it follows from (5.23) and Lemma 5.10 that

$$
\begin{aligned}
(\star) \leq & (N h)^{|\alpha|} \cdot|\alpha| ! \cdot \sum \frac{1}{k_{1} ! \cdot \ldots \cdot k_{\ell} !} \cdot[C(1+|\eta|)]^{\left(k_{1}+\ldots+k_{\ell}\right)} \cdot m_{\left|\delta_{1}\right|}^{k_{1}} \times \\
& \ldots \times m_{\left|\delta_{\ell}\right|}^{k_{\ell}} \cdot \inf _{p \in \mathbf{N}_{0}}\left(\frac{m_{p} \cdot p !}{\varepsilon^{p} \cdot(1+|\eta|)^{p}}\right) \\
\leq & (N h)^{|\alpha|}|\alpha| ! \sum \frac{1}{k_{1} ! \cdot \ldots \cdot k_{\ell} !}[C(1+|\eta|)]^{\left(k_{1}+\ldots+k_{\ell}\right)} m_{\left|\delta_{1}\right|}^{k_{1}} \times \\
& \ldots \times m_{\left|\delta_{\ell}\right|}^{k_{\ell}} \cdot \frac{m_{\left(k_{1}+\ldots+k_{\ell}\right)}\left(k_{1}+\ldots+k_{\ell}\right) !}{\varepsilon^{\left(k_{1}+\ldots+k_{\ell}\right)}(1+|\eta|)^{\left(k_{1}+\ldots+k_{\ell}\right)}} \\
\leq & (N h)^{|\alpha|}|\alpha| ! \cdot \sum \frac{\left(k_{1}+\ldots+k_{\ell}\right) !}{k_{1} ! \cdot \ldots \cdot k_{\ell} !}\left(\frac{C}{\varepsilon}\right)^{\left(k_{1}+\ldots+k_{\ell}\right)} \times \\
& \times\left(m_{\left(k_{1}+\ldots+k_{\ell}\right)} \cdot m_{\left|\delta_{1}\right|}^{k_{1}} \cdot \ldots \cdot m_{\left|\delta_{\ell}\right|}^{k_{\ell}}\right) \\
\leq & (N h)^{|\alpha|} \cdot m_{|\alpha|} \cdot|\alpha| ! \cdot \underbrace{\sum \frac{\left(k_{1}+\ldots+k_{\ell}\right) !}{k_{1} ! \cdot \ldots \cdot k_{\ell} !}\left(\frac{C}{\varepsilon}\right)^{\left(k_{1}+\ldots+k_{\ell}\right)}}_{(\triangle)} .
\end{aligned}
$$

As consequence of Lemma 4.8 of [BM], there exist $M, \lambda>0$ depending on $C$ and $\varepsilon$ such that

$$
(\triangle) \leq M \cdot \lambda^{|\alpha|}
$$

Therefore it is sufficient to take $C_{\varepsilon}=M$ and $h_{\varepsilon}=N \cdot h \cdot \lambda$ to finalize the proof.

Theorem 5.12. Let $T: \mathscr{D}_{\mathscr{M}}^{\prime}\left(\mathbf{T}^{N+1}\right) \rightarrow \mathscr{D}_{\mathscr{M}}^{\prime}\left(\mathbf{T}^{N+1}\right)$ be the operator given by

$$
T\left(\sum_{\eta \in \mathbf{Z}} \widehat{u}(t, \eta) e^{i x \eta}\right)=\sum_{\eta \in \mathbf{Z}} \widehat{u}(t, \eta) e^{i(A(t)+x) \eta} .
$$

Then $T$ is an automorphism. Furthermore, the same holds for $\left.T\right|_{\mathcal{E}_{\mathscr{M}}\left(\mathbf{T}^{N+1}\right)}$. 
Proof. We begin by verifying that $T$ is well defined. By Theorem 4.18, given $\varepsilon, h>0$, one has to find $C_{\varepsilon, h}>0$ such that $\left|\left\langle\widehat{u}(t, \eta) e^{i \eta A(t)}, \varphi\right\rangle\right| \leq C_{\varepsilon, h} \cdot\|\varphi\|_{\mathscr{M}, h} \cdot \sup _{n \in \mathbf{N}_{0}}\left(\frac{\varepsilon^{n} \cdot(1+|\eta|)^{n}}{m_{n} \cdot n !}\right), \quad \forall \eta \in \mathbf{Z}, \quad \forall \varphi \in \mathcal{E}_{\mathscr{M}, h}\left(\mathbf{T}^{N}\right)$.

Fix $\varphi \in \mathcal{E}_{\mathscr{M}, h}\left(\mathbf{T}^{N}\right)$; for $\delta>0$ that will be chosen later, we denote

$$
(*)=\inf _{p \in \mathbf{N}_{0}}\left(\frac{m_{p} \cdot p !}{\delta^{p} \cdot(1+|\eta|)^{p}}\right) \cdot\left|\partial_{t}^{\alpha}\left(e^{i \eta A(t)} \cdot \varphi(t)\right)\right| .
$$

It follows from Proposition 5.11 that

$$
\begin{aligned}
(*) & \leq \sum_{\beta \leq \alpha}\left(\begin{array}{c}
\alpha \\
\beta
\end{array}\right)\left(C_{\delta} \cdot h_{\delta}^{|\beta|} \cdot m_{|\beta|} \cdot|\beta| !\right) \cdot\left(\|\varphi\|_{\mathscr{M}, h} \cdot h^{|\alpha|-|\beta|} \cdot m_{|\alpha|-|\beta|} \cdot(|\alpha|-|\beta|) !\right) \\
& \leq C_{\delta} \cdot\|\varphi\|_{\mathscr{M}, h} \cdot h_{\delta}^{|\alpha|} \cdot m_{|\alpha|} \cdot|\alpha| !,
\end{aligned}
$$

where $h_{\delta}^{\prime}=2 \cdot \max \left\{h_{\delta}, h\right\}$. Hence

$$
\inf _{p \in \mathbf{N}_{0}}\left(\frac{m_{p} \cdot p !}{\delta^{p} \cdot(1+|\eta|)^{p}}\right) \cdot\left|\partial_{t}^{\alpha}\left(e^{i \eta A(t)} \cdot \varphi(t)\right)\right| \leq C_{\delta} \cdot\|\varphi\|_{\mathscr{M}, h} \cdot h_{\delta}^{\prime|\alpha|} \cdot m_{\alpha} \cdot \alpha ! .
$$

On the other hand, by Theorem 3.4, for every $\rho>0$ there exists $C_{\rho}>0$ such that

$$
|\langle u, f\rangle| \leq C_{\rho} \cdot \sup _{\gamma \in \mathbf{N}_{0}^{N+1}}\left(\frac{\left\|\partial^{\gamma} f\right\|_{\infty} \cdot \rho^{|\gamma|}}{m_{|\gamma|} \cdot|\gamma| !}\right), \quad \forall f \in \mathcal{E}_{\mathscr{M}}\left(\mathbf{T}^{N+1}\right),
$$

which implies that

$$
\begin{aligned}
& \left|\left\langle\widehat{u}(t, \eta) e^{i \eta A(t)}, \varphi(t)\right\rangle\right| \\
& \leq \frac{C_{\rho}}{2 \pi} \cdot \sup _{\left(\gamma_{1}, \gamma_{2}\right) \in \mathbf{N}_{0}^{N} \times \mathbf{N}_{0}}\left(\frac{\left\|\partial_{t}^{\gamma_{1}}\left(e^{i \eta A(t)} \varphi(t)\right)\right\|_{\infty} \cdot\left\|\partial_{x}^{\gamma_{2}}\left(e^{-i x \eta}\right)\right\|_{\infty} \cdot \rho^{\left|\gamma_{1}\right|+\left|\gamma_{2}\right|}}{m_{\left|\gamma_{1}\right|+\left|\gamma_{2}\right|} \cdot\left(\left|\gamma_{1}\right|+\left|\gamma_{2}\right|\right) !}\right) \\
& \leq \frac{C_{\rho}}{2 \pi} \cdot \sup _{\left(\gamma_{1}, \gamma_{2}\right) \in \mathbf{N}_{0}^{N} \times \mathbf{N}_{0}}\left(\frac{\left\|\partial_{t}^{\gamma_{1}}\left(e^{i \eta A(t)} \varphi(t)\right)\right\|_{\infty} \cdot(1+|\eta|)^{\left|\gamma_{2}\right|} \cdot \rho^{\left|\gamma_{1}\right|+\left|\gamma_{2}\right|}}{m_{\left|\gamma_{1}\right|+\left|\gamma_{2}\right|} \cdot\left(\left|\gamma_{1}\right|+\left|\gamma_{2}\right|\right) !}\right) .
\end{aligned}
$$

Let $(\dagger):=\left\|\partial_{t}^{\gamma_{1}}\left(e^{i \eta A(t)} \varphi(t)\right)\right\|_{\infty} \cdot(1+|\eta|)^{\left|\gamma_{2}\right|} \cdot \rho^{\left|\gamma_{1}\right|+\left|\gamma_{2}\right|}$; if $\delta=\frac{\varepsilon}{2 H}$, it follows from Lemma 4.17 and (5.25) that

$$
\begin{aligned}
(\dagger) \leq & \left\|\partial_{t}^{\gamma_{1}}\left(e^{i A(t) \eta} \varphi(t)\right)\right\|_{\infty} \cdot \inf _{p \in \mathbf{N}_{0}}\left(\frac{m_{p} \cdot p !}{\delta^{p} \cdot(1+|\eta|)^{p}}\right)^{2} \\
& \cdot(1+|\eta|)^{\left|\gamma_{2}\right|} \cdot \sup _{n \in \mathbf{N}_{0}}\left(\frac{\varepsilon^{n} \cdot(1+|\eta|)^{n}}{m_{n} \cdot n !}\right) \cdot \rho^{\left|\gamma_{1}\right|+\left|\gamma_{2}\right|} \\
\leq & C_{\delta} \cdot\|\varphi\|_{\mathscr{M}, h} \cdot h_{\delta}^{\prime\left|\gamma_{1}\right|} \cdot m_{\left|\gamma_{1}\right|} \cdot\left|\gamma_{1}\right| ! \cdot\left(\frac{m_{\left|\gamma_{2}\right|} \cdot\left|\gamma_{2}\right| ! \cdot(2 H)^{\left|\gamma_{2}\right|}}{\varepsilon^{\left|\gamma_{2}\right|}}\right) \\
& \cdot \sup _{n \in \mathbf{N}_{0}}\left(\frac{\varepsilon^{n} \cdot(1+|\eta|)^{n}}{m_{n} \cdot n !}\right) \cdot \rho^{\left|\gamma_{1}\right|+\left|\gamma_{2}\right|} \\
\leq & C_{\delta} \cdot\|\varphi\|_{\mathscr{M}, h} \cdot\left(\frac{h_{\delta}^{\prime} \cdot 2 H \cdot \rho}{\varepsilon}\right)^{\left|\gamma_{1}\right|+\left|\gamma_{2}\right|} \\
& \cdot m_{\left|\gamma_{1}\right|+\left|\gamma_{2}\right|} \cdot\left(\left|\gamma_{1}\right|+\left|\gamma_{2}\right|\right) ! \cdot\left(\sup _{n \in \mathbf{N}_{0}} \frac{\varepsilon^{n} \cdot(1+|\eta|)^{n}}{m_{n} \cdot n !}\right),
\end{aligned}
$$


since we may suppose $\varepsilon<2 H$. By taking $\rho=\frac{\varepsilon}{h_{\delta}^{\prime} \cdot 2 H}$ and applying (5.26) and (5.27), we obtain

$$
\left|\left\langle\widehat{u}(t, \eta) e^{i \eta A(t)}, \varphi(t)\right\rangle\right| \leq \frac{C_{\rho}}{2 \pi} \cdot C_{\delta} \cdot\|\varphi\|_{\mathscr{M}, h} \cdot \sup _{n \in \mathbf{N}_{0}}\left(\frac{\varepsilon^{n} \cdot(1+|\eta|)^{n}}{m_{n} \cdot n !}\right) .
$$

Note that both $\rho$ and $\delta$ depend only on $\varepsilon, h$ and thus first part of the proof is complete.

Now let $\psi \in \mathcal{E}_{\mathscr{M}}\left(\mathbf{T}^{N+1}\right)$; by Theorem 4.12 , there exist $C, h, \delta>0$ such that

$$
\left|\partial_{t}^{\beta} \widehat{\psi}(t, \eta)\right| \leq C \cdot h^{|\beta|} \cdot m_{|\beta|} \cdot|\beta| ! \cdot \inf _{p \in \mathbf{N}_{0}}\left(\frac{m_{p} \cdot p !}{\delta^{p} \cdot(1+|\eta|)^{p}}\right), \quad \forall t \in \mathbf{T}, \quad \forall \beta \in \mathbf{N}_{0}^{N},
$$

which implies that

$$
\begin{aligned}
& \left|\partial_{t}^{\alpha}\left(\widehat{\psi}(t, \eta) \cdot e^{i A(t) \eta}\right)\right| \\
& \leq \sum_{\beta \leq \alpha}\left(\begin{array}{c}
\alpha \\
\beta
\end{array}\right) C \cdot h^{|\beta|} \cdot m_{|\beta|} \cdot|\beta| ! \cdot \inf _{p \in \mathbf{N}_{0}}\left(\frac{m_{p} \cdot p !}{\delta^{p} \cdot(1+|\eta|)^{p}}\right) \cdot\left|\partial_{t}^{\alpha-\beta}\left(e^{i A(t) \eta}\right)\right|
\end{aligned}
$$

We apply once again Lemma 4.17 and (5.25):

$$
\begin{aligned}
& \inf _{p \in \mathbf{N}_{0}}\left(\frac{m_{p} \cdot p !}{\delta^{p} \cdot(1+|\eta|)^{p}}\right)\left|\partial_{t}^{\alpha-\beta}\left(e^{i A(t) \eta}\right)\right| \\
& \leq C_{1} \inf _{p \in \mathbf{N}_{0}}\left(\frac{(2 H)^{p} \cdot m_{p} \cdot p !}{\delta^{p} \cdot(1+|\eta|)^{p}}\right) \cdot h_{1}^{|\alpha|-|\beta|} \cdot m_{|\alpha|-|\beta|} \cdot(|\alpha|-|\beta|) ! .
\end{aligned}
$$

By associating (5.28) to (5.29), setting $C_{2}=C C_{1}$ and $h_{2}=2 \max \left\{h_{1}, h\right\}$, we deduce that

$$
\left|\partial_{t}^{\alpha}\left(\widehat{\psi}(t, \eta) \cdot e^{i A(t) \eta}\right)\right| \leq C_{2} \cdot h_{2}^{|\alpha|} \cdot m_{|\alpha|} \cdot|\alpha| ! \cdot \inf _{p \in \mathbf{N}_{0}}\left(\frac{(2 H)^{p} \cdot m_{p} \cdot p !}{\delta^{p} \cdot(1+|\eta|)^{p}}\right) .
$$

Therefore $T(\psi) \in \mathcal{E}_{\mathscr{M}}\left(\mathbf{T}^{N}\right)$, by Theorem 4.12 .

To see that $T$ is an automorphism, it is easy to check that same properties proved for $T$ also hold up for $T^{\prime}: \mathscr{D}_{\mathscr{M}}^{\prime}\left(\mathbf{T}^{N+1}\right) \rightarrow \mathscr{D}_{\mathscr{M}}^{\prime}\left(\mathbf{T}^{N+1}\right)$ given by

$$
T^{\prime}\left(\sum_{\eta \in \mathbf{Z}} \widehat{u}(t, \eta) e^{i x \eta}\right)=\sum_{\eta \in \mathbf{Z}} \widehat{u}(t, \eta) e^{i(-A(t)+x) \eta} .
$$

Moreover, $T^{\prime}$ is the inverse of $T$.

Theorem 5.13. The system of real vector fields $\mathcal{L}$ described in (5.17) is globally $\mathscr{M}$-hypoelliptic if and only if the same is valid for the system with constant coefficients $\widetilde{\mathcal{L}}$ defined in (5.20). $T^{-1}$.

Proof. It follows immediately from Theorem 5.12 and the fact that $\tilde{L}_{j}=T \circ L_{j} \circ$

Corollary 5.14. Let $\mathcal{P}$ be a system of $N$ vector fields acting on $\mathbf{T}_{t}^{N} \times \mathbf{T}_{x}$ given by

$$
P_{j}=\frac{\partial}{\partial t_{j}}+b_{j}\left(t_{j}\right) \frac{\partial}{\partial x}, \quad j=1,2, \ldots, N,
$$

where each $b_{j} \in \mathcal{E}_{\mathscr{M}}(\mathbf{T} ; \mathbf{R})$. Then $\mathcal{P}$ is globally $\mathscr{M}$-hypoelliptic if and only if the same holds for $\widetilde{\mathcal{P}}$, whose equations are

$$
\widetilde{P}_{j}=\frac{\partial}{\partial t_{j}}+b_{j_{0}} \frac{\partial}{\partial x}, \quad j=1,2, \ldots, N,
$$


and $b_{j_{0}}=\frac{1}{2 \pi} \int_{0}^{2 \pi} b_{j}\left(t_{j}\right) d t_{j}$.

Proof. It follows from the fact that the functions $b_{j}$ trivially satisfy (5.18).

Acknowledgements. The author would like to thank Prof. Alexandre Kirilov for his suggestions and Prof. Gerson Petronilho for his notes on Gevrey ultradistributions. Both were really important for the development of the present work.

\section{References}

[AJ] Albanese, A. A., and D. Jornet: Global regularity in ultradifferentiable classes. - Ann. Mat. Pura Appl. (4) 193:2, 2014, 369-387.

[AP] Albanese, A. A., and P. Popivanov: Global analytic and Gevrey solvability of sublaplacians under Diophantine conditions. - Ann. Mat. Pura Appl. (4) 185:3, 2006, 395-409.

[AZ] Albanese, A. A., and L. Zanghirati: Global hypoellipticity and global solvability in Gevrey classes on the $n$-dimensional torus. - J. Differential Equations 199:2, 2004, 256-268.

[Ber] Bergamasco, A. P.: Remarks about global analytic hypoellipticity. - Trans. Amer. Math. Soc. 351:10, 1999, 4113-4126.

[BCM] Bergamasco, A. P., P. D. Cordaro, and P. A. Malagutti: Globally hypoelliptic systems of vector fields. - J. Funct. Anal. 114:2, 1993, 267-285.

[BCP] Bergamasco, A., P.D. Cordaro, and G. Petronilho: Global solvability for a class of complex vector fields on the two-torus. - Comm. Partial Differential Equations 29:5-6, 2004, 785-819.

[BP] Bergamasco, A.P., and G. Petronilho: Global solvability of a class of involutive systems. - J. Math. Anal. Appl. 233:1, 1999, 314-327.

[BGS] Bergamasco, A., P.D. Da Silva, and R. B. Gonzalez: Global solvability and global hypoellipticity in Gevrey classes for vector fields on the torus. - J. Differential Equations $264: 5,2018,3500-3526$.

[BM] Bierstone, E., and P.D. Milman: Resolution of singularities in Denjoy-Carleman classes. - Selecta Math. (N.S.) 10:1, 2004, 1.

[BMM] Bonet, J., R. Meise, and S. N. Melikhov: A comparison of two different ways to define classes of ultradifferentiable functions. - Bull. Belg. Math. Soc. Simon Stevin. 14:3, 2004, $425-444$.

[BMT] Braun, R. W., R. Meise, and B. A. TAYlor: Ultradifferentiable functions and Fourier analysis. - Results Math. 17:3-4, 1990, 206-237.

[BCCJ] Braun Rodrigues, N., G. Chinni, P. Cordaro, and M. Jahnke: Lower order perturbation and global analytic vectors for a class of globally analytic hypoelliptic operators. Proc. Amer. Math. Soc. 144:12, 2016, 5159-5170.

[Car] Carleman, T.: Les fonctions quasi analytiques: leçons professées au Collège de France. Gauthier-Villars et Cie, 1926.

[CC $]$ Chinni, G., and P.D. Cordaro: On global analytic and Gevrey hypoellipticity on the torus and the Métivier inequality. - Comm. Partial Differential Equations 42, 2017, 121-141.

[Den] Denjoy, A.: Sur les fonctions quasi-analytiques de variable rélle. - C. R. Acad. Sci. Paris 173, 1921, 1320-1322.

[DGY] Dickinson, D., T. Gramchev, and M. Yoshino: Perturbations of vector fields on tori: resonant normal forms and Diophantine phenomena. - Proc. Edinb. Math. Soc. (2) 45:3, $2002,731-759$.

[GPY] Gamchev, T., P. Popivanov, and M. Yoshino: Global properties in spaces of generalized functions on the torus for second order differential operators with variable coefficients.

- Rend. Sem. Mat. Univ. Pol. Torino 51:2, 1993, 144-174. 
[Gr] Greenfield, S. J.: Hypoelliptic vector fields and continued fractions. - Proc. Amer. Math. Soc. 31:1, 1972, 115-118.

[GW] Greenfield, S. J., and N. R. Wallach: Global hypoellipticity and Liouville numbers. Proc. Amer. Math. Soc. 31:1, 1972, 112-114.

[HW] Hardy, G.H., and E. M.: An introduction to the theory of numbers. - Oxford Univ. Press, 1979.

[Him] Himonas, A.: Global analytic and Gevrey hypoellipticity of sublaplacians under Diophantine conditions. - Proc. Amer. Math. Soc. 129:7, 2001, 2061-2067.

[HP] Himonas, A. A., and G. Petronilho: On Gevrey regularity of globally $C^{\infty}$ hypoelliptic operators. - J. Differential Equations 207:2, 2004, 267-284.

[Hor] HÖRMANDER, L.: The analysis of linear partial differential operators I: Distribution theory and Fourier analysis. - Springer, 2015.

[Hou] Hounie, J.: Globally hypoelliptic and globally solvable first-order evolution equations. Trans. Amer. Math. Soc. 252, 1979, 233-248.

[JKM] Junior, A. A., A. Kirilov, and C. DE Medeira: Global Gevrey hypoellipticity on the torus for a class of systems of complex vector fields. - J. Math. Anal. Appl. 474:1, 2019, $712-732$.

[K2] Komatsu, H.: Projective and injective limits of weakly compact sequences of locally convex spaces. - J. Math. Soc. Japan 19, 1967, 366-383.

[K1] Komatsu, H.: Ultradistribution I. Structure theorems and a characterization. - J. Fac. Sci. Tokyo Ser. IA 20, 1973, 25-105.

[PZ] Petronilho, G., and S. L. Zani: Global $s$-solvability and global $s$-hypoellipticity for certain perturbations of zero order of systems of constant real vector fields. - J. Differential Equations 244:9, 2008, 2372-2403.

[RudI] Rudin, W.: Division in algebras of infinitely differentiable functions. - J. Math. Mech. 11:5, 1962, 797-809.

[RudII] Rudin, W.: Real and complex analysis. - Tata McGraw-Hill Education, 2006.

[Tar] TARTAKOFF, D.: Global (and local) analyticity for second order operators constructed from rigid vector fields on products of tori. - Trans. Amer. Math. Soc. 348:7, 1996, 2577-2583.

[Th] Thilliez, V.: On quasianalytic local rings. - Expo. Math. 26:1, 2008, 1-23.

[WC] Wenyi, C., and M. Y. Chi: Hypoelliptic vector fields and almost periodic motions on the torus $T^{n}$. - Comm. Partial Differential Equations 25:1-2, 2000, 337-354.

Received 4 August $2020 \bullet$ Accepted 8 December 2020 